\title{
Inelastic scattering in resonant tunneling
}

\section{Wingreen, Ned S.; Jacobsen, Karsten Wedel; Wilkins, John W.}

\section{Published in:}

Physical Review B

Link to article, DOI:

10.1103/PhysRevB.40.11834

Publication date:

1989

\section{Document Version}

Publisher's PDF, also known as Version of record

Link back to DTU Orbit

Citation (APA):

Wingreen, N. S., Jacobsen, K. W., \& Wilkins, J. W. (1989). Inelastic scattering in resonant tunneling. Physical Review B, 40(17), 11834-11850. https://doi.org/10.1103/PhysRevB.40.11834

\section{General rights}

Copyright and moral rights for the publications made accessible in the public portal are retained by the authors and/or other copyright owners and it is a condition of accessing publications that users recognise and abide by the legal requirements associated with these rights.

- Users may download and print one copy of any publication from the public portal for the purpose of private study or research.

- You may not further distribute the material or use it for any profit-making activity or commercial gain

- You may freely distribute the URL identifying the publication in the public portal

If you believe that this document breaches copyright please contact us providing details, and we will remove access to the work immediately and investigate your claim 


\title{
Inelastic scattering in resonant tunneling
}

\author{
Ned S. Wingreen* \\ Laboratory of Atomic and Solid State Physics, Cornell University, Ithaca, New York 14853-2501 \\ Karsten W. Jacobsen \\ Laboratory of Applied Physics, Technical University of Denmark, DK-2800 Lyngby, Denmark \\ John W. Wilkins \\ Department of Physics, Ohio State University, 174 West 18th Avenue, Columbus, Ohio 43210-1106
}

(Received 1 May 1989; revised manuscript received 10 August 1989)

\begin{abstract}
The exact resonant-tunneling transmission probability for an electron interacting with phonons is presented in the limit that the elastic coupling to the leads is independent of energy. The phonons produce transmission sidebands but do not affect the integrated transmission probability or the escape rate of the electron from the resonant site. In the Appendixes, we evaluate the Green function that appears in the expression for the transmission probability.
\end{abstract}

\section{INTRODUCTION}

The understanding of transport in small semiconductor structures relies on the assumption of transmission between ideal leads. This approximation has allowed the proper identification of the symmetries of transport coefficients in a magnetic field, ${ }^{1,2}$ quantitative analysis of four-probe conductance fluctuations, ${ }^{3-6}$ and explanations of the quantization of conductance through a constriction $^{7-12}$ and of the quenching of the Hall effect in narrow samples. ${ }^{13,14}$ At the heart of the ideal lead description is the belief that when an electron enters a lead, it loses phase coherence so that transport can be described entirely by transmission and reflection probabilities between leads. However, a complete microscopic description of this phase randomization does not exist.

To address inelastic scattering in transport from a fundamental point of view we study a model problem: resonant tunneling through a single site coupled to phonons. While inelastic scattering in resonant tunneling has been addressed before for tunneling through states localized by disorder ${ }^{15}$ and for tunneling through a double-barrier heterostructure $^{16,17}$ (see Fig. 1), all these previous approaches were phenomenological, with inelastic scattering included as an effective absorption coefficient. In a recent paper, ${ }^{18}$ we introduced a Hamiltonian formulation for resonant tunneling with the electron-phonon interaction included on a microscopic footing, and presented an exact solution for the phonon-assisted transmission probability. Here we explain in detail how resonant tunneling with inelastic scattering can be addressed and solved as a scattering problem. First, the transmission probabilities are expressed in terms of a two-particle Green function, which factors into two one-particle Green functions in the case of no electron-phonon interaction. Second, we find an exact solution for the two-particle Green function provided the elastic couplings to the leads-roughly the density of states in each lead multiplied by the square of the tunneling matrix elements through the barriers-are independent of energy. Since the density of states in the leads will be constant if the band is broad, we refer to the limit of energy-independent couplings as the wide-band limit.

From the exact solution in the wide-band limit, we obtain two sum rules restricting $T_{\text {tot }}(\varepsilon)$ the total transmission probability in the presence of phonons: (1) the integral of $T_{\text {tot }}(\varepsilon)$ is independent of phonons so that the total transmissivity of the structure is conserved, and (2) the first energy moment of $T_{\text {tot }}(\varepsilon)$ is also conserved, so that the center of the transmission spectrum remains fixed. Importantly, the escape rate of an electron placed on the resonant site is also independent of the interaction with phonons. Insofar as the tunneling delay time and the escape time are the same, ${ }^{19}$ this implies that phonon

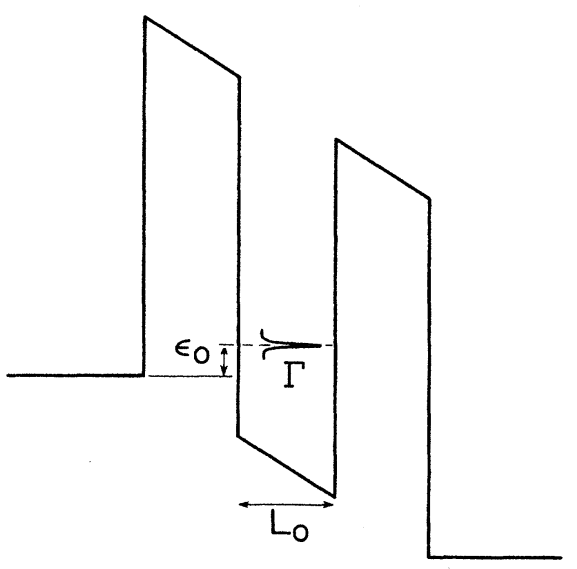

FIG. 1. Schematic drawing of a resonant-tunneling structure under bias. The quantum well of width $L_{0}$ has a quasibound state at energy $\varepsilon_{0}$. In the absence of electron-phonon interactions, the probability for an electron with energy near $\varepsilon_{0}$ to tunnel through the structure is a Lorentzian of width $\Gamma$ as a function of the electron's incident energy. 
broadening of the transmission resonance will not enhance the speed of tunneling.

The same model Hamiltonian for resonant tunneling with phonons has been studied independently by two other groups in addition to ourselves. Glazman and Shekter ${ }^{20,21}$ obtained a solution identical to ours via an equation of motion method, and Jonson, ${ }^{22}$ using the tunneling Hamiltonian formalism, obtained a solution to first order in the tunneling strength. While the results are in agreement, the exact representation of the transmission probability in terms of a Green function is unique to us. We believe this Green-function approach is easier to generalize to treat structures with more than one electronic state than either the equation of motion method or the tunneling Hamiltonian approach.

This paper is organized as follows. In Sec. II, we present the Hamiltonian for resonant tunneling through a single site coupled to phonons and relate the transmission probability to a two-particle Green function via the $S$ matrix formalism. An exact solution for the Green function in the wide-band limit is obtained in Sec. III, and sum rules governing the total transmission probability are derived and illustrated. Section IV is devoted to a brief proof that the escape rate from the quantum well is independent of the interaction with phonons. In Sec. V, we apply the results for the transmission probability to calculate the current through a quasi-one-dimensional resonant-tunneling structure, including an interaction with polar-optical phonons. We generalize our results to three dimensions in Sec. VI, and calculate the current for a range of couplings to optical phonons in Sec. VII. Section VIII contains our conclusions.

\section{TRANSMISSION PROBABILITY OF AN ELECTRON COUPLED TO PHONONS}

The Hamiltonian we use to model resonant tunneling is the sum of an electron term $H_{e}$, a phonon term $H_{\mathrm{ph}}$, and an electron-phonon interaction term $H_{\text {int }}$. The electron Hamiltonian,

$$
\begin{aligned}
H_{e}= & \varepsilon_{0} c^{\dagger} c+\sum_{k} \varepsilon_{k L} c_{k L}^{\dagger} c_{k L}+\sum_{k} \varepsilon_{k R} c_{k R}^{\dagger} c_{k R} \\
& +\sum_{k} V_{k L}\left(c_{k L}^{\dagger} c+c^{\dagger} c_{k L}\right)+\sum_{k} V_{k R}\left(c_{k R}^{\dagger} c+c^{\dagger} c_{k R}\right)
\end{aligned}
$$

describes a single state of energy $\varepsilon_{0}$ (e.g., the ground state of a quantum well) coupled by hopping matrix elements $V_{k L}$ and $V_{k R}$ to states of energy $\varepsilon_{k L}$ and $\varepsilon_{k R}$ in ideal leads on its left and right, respectively. As is usual in the description of transport in small structures, the leads are referred to interchangeably as "contacts," with subscript $L$ denoting the left contact and subscript $R$ denoting the right contact. The phonon Hamiltonian is just a sum of harmonic oscillators,

$$
H_{\mathrm{ph}}=\sum_{\mathrm{q}} \hbar \omega_{\mathrm{q}} a_{\mathrm{q}}^{\dagger} a_{\mathrm{q}}
$$

and the electron-phonon interaction is restricted to the resonant site

$$
H_{\text {int }}=c^{\dagger} c \sum_{\mathrm{q}} M_{\mathrm{q}}\left(a_{\mathrm{q}}^{\dagger}+a_{-\mathrm{q}}\right),
$$

where $c^{\dagger}$ and $c$ are the Fermion operators for the electron on the resonant site.

Central to our analysis of the transport properties of the model is the noninteracting, retarded Green function of the resonant site,

$$
G_{R}^{0}(t)=-i \Theta(t)\left\langle 0\left|c(t) c^{\dagger}(0)\right| 0\right\rangle,
$$

where

$$
c(t)=e^{i H_{e} t / \hbar} c(0) e^{-i H_{e} t / \hbar},
$$

and $|0\rangle$ denotes the electron vacuum. Solving the electronic Hamiltonian is simpler in terms of the Fourier transform of the Green function,

$$
G_{R}^{0}(\varepsilon)=\int_{-\infty}^{\infty} \frac{d t}{\hbar} e^{i \varepsilon t / \hbar} G_{R}^{0}(t)
$$

If we treat the tunneling between the resonant site and the contacts as a perturbation on the bare Hamiltonian, we find from the Dyson equation ${ }^{23}$

$$
G_{R}^{0}(\varepsilon)=\frac{1}{\varepsilon-\varepsilon_{0}-\Sigma_{R}(\varepsilon)+i \Gamma(\varepsilon) / 2},
$$

where the elastic coupling to the leads $\Gamma(\varepsilon)=\Gamma_{L}(\varepsilon)$ $+\Gamma_{R}(\varepsilon)$ depends on the hopping strength and the contact density of states according to

$$
\Gamma_{L(R)}(\varepsilon)=2 \pi \sum_{k}\left|V_{k L(k R)}\right|^{2} \delta\left(\varepsilon-\varepsilon_{k L(k R)}\right) .
$$

The real shift in the energy of the resonance,

$$
\Sigma_{R}(\varepsilon)=\int \frac{d \varepsilon^{\prime}}{2 \pi} \frac{\Gamma(\varepsilon)}{\varepsilon-\varepsilon^{\prime}},
$$

is the Hilbert transform of the elastic coupling $\Gamma(\varepsilon)$.

In the wide-band limit, in which the bandwidth in the contacts is much larger than both the resonance width and the phonon energies, the contact densities of states

$$
\rho_{L(R)}(\varepsilon)=\sum_{k} \delta\left(\varepsilon-\varepsilon_{k L(k R)}\right)
$$

will be constant in the region of the resonance. If the hopping matrix elements also vary slowly with energy, the couplings to the contacts $\Gamma_{L}$ and $\Gamma_{R}$ will be constant,

$$
\Gamma_{L(R)}=2 \pi\left|V_{L(R)}\right|^{2} \rho_{L(R)}\left(\varepsilon_{0}\right) .
$$

In this special case, $\Gamma(\varepsilon)$ is independent of energy and the Green function in Eq. (4) reduces to a pure exponential

$$
G_{R}^{0}(t)=-i \Theta(t) e^{\left(-i \varepsilon_{0}-\Gamma / 2\right) t / \hbar} .
$$

The special virtue of the wide-band limit is that the exponential form of $G_{R}^{0}(t)$ permits us to obtain an exact solution for the transmission probability including the interaction with phonons.

The transmission matrix. We will calculate the probability $T\left(\varepsilon^{\prime}, \varepsilon\right)$ that an electron with energy $\varepsilon$ incident from the left lead will be transmitted to the right lead with energy $\varepsilon^{\prime}$, the difference in energy, $\varepsilon-\varepsilon^{\prime}$, being 
"left" in the phonon system. $T\left(\varepsilon^{\prime}, \varepsilon\right)$ is referred to as the transmission matrix as it is a function of both the incoming and outgoing energies of the electron. Because the electron-phonon interaction is confined to a finite region of space, we can apply the $S$-matrix scattering formal- ism ${ }^{24}$ to evaluate the transmission matrix $T\left(\varepsilon^{\prime}, \varepsilon\right)$. We find (Appendix A) that $T\left(\varepsilon^{\prime}, \varepsilon\right)$ is given exactly by the product of the elastic couplings to the two leads and the Fourier transform of a Green function for the resonant level,

$$
T\left(\varepsilon^{\prime}, \varepsilon\right)=\Gamma_{L}(\varepsilon) \Gamma_{R}\left(\varepsilon^{\prime}\right) \iiint \frac{d \tau d s d t}{2 \pi \hbar^{3}} e^{i\left[\left(\varepsilon-\varepsilon^{\prime}\right) \tau+\varepsilon^{\prime} t-\varepsilon s\right] / \hbar} G(\tau, s, t)
$$

The transmission Green function in Eq. (13),

$$
G(\tau, s, t)=\Theta(s) \Theta(t)\left\langle c(\tau-s) c^{\dagger}(\tau) c(t) c^{\dagger}(0)\right\rangle,
$$

is evaluated on a state with no electron present, but with a thermal ensemble of the phonons. The electron operators are in the Heisenberg representation,

$$
c(t)=e^{i H t / \hbar} c(0) e^{-i H t / \hbar},
$$

and contain the full electron-phonon interaction.

It is important to note that Eq. (13) applies in both the "coherent" limit, in which there is no electron-phonon interaction, and in the "incoherent" limit of strong interaction where the probability of inelastic scattering is high. The factors of $\Gamma(\varepsilon)$ and $\Gamma\left(\varepsilon^{\prime}\right)$ represent the tunneling strength of the incoming and outgoing electron through the barriers, while the Green function $G(\tau, s, t)$ includes all the dynamics, including the existence of the resonant level. In the absence of electron-phonon interaction the transmission Green function reduces to the product of two single-particle Green functions,

$$
G_{0}(\tau, s, t)=G_{R}^{0}(t)\left[G_{R}^{0}(s)\right]^{*},
$$

and the noninteracting transmission matrix is given by

$$
T^{0}\left(\varepsilon^{\prime}, \varepsilon\right)=\frac{\Gamma_{L}(\varepsilon) \Gamma_{R}\left(\varepsilon^{\prime}\right) \delta\left(\varepsilon-\varepsilon^{\prime}\right)}{\left[\varepsilon-\varepsilon_{0}-\Sigma_{R}(\varepsilon)\right]^{2}+[\Gamma(\varepsilon) / 2]^{2}}
$$

The peak shape of the transmission probability $T^{0}\left(\varepsilon^{\prime}, \varepsilon\right)$ will be Lorentzian as long as the resonance shift $\Sigma_{R}(\varepsilon)$ and the elastic coupling $\Gamma(\varepsilon)$ are not strongly energy dependent, in which case $\Gamma\left(\varepsilon_{0}\right)$ can be identified as the elastic resonance width.

From the $S$-matrix calculation, one can also obtain an expression for the interacting reflection matrix, describing the probability a particle incident with energy $\varepsilon$ will be reflected with energy $\varepsilon^{\prime}$. As shown in Appendix A, Eq. (A19),

$$
\begin{aligned}
R\left(\varepsilon^{\prime}, \varepsilon\right)= & \frac{\Gamma_{L}\left(\varepsilon^{\prime}\right) T\left(\varepsilon^{\prime}, \varepsilon\right)}{\Gamma_{R}\left(\varepsilon^{\prime}\right)} \\
& +\delta\left(\varepsilon-\varepsilon^{\prime}\right)\left\{1+2 \Gamma_{L}(\varepsilon) \operatorname{Im}\left[G_{R}(\varepsilon)\right]\right\},
\end{aligned}
$$

where $G_{R}(\varepsilon)$ is the Green function of the resonant site including the electron-phonon interaction.

\section{EXACT SOLUTION IN THE WIDE-BAND LIMIT}

The problem of evaluating the Green function of a single site coupled to phonons is well known in the context of core-level x-ray emission. ${ }^{25-28}$ In the case of energyindependent couplings, $\Gamma_{L}$ and $\Gamma_{R}$, the techniques developed to treat the interaction of a stationary core hole with phonons can be adapted directly to evaluate the transmission Green function $G(\tau, s, t)$ defined in Eq. (14). The restriction to constant couplings is necessary so that the noninteracting Green function in Eq. (4) is a pure exponential, $G_{R}^{0}(t)=-i \Theta(t) \exp \left[\left(-i \varepsilon_{0}-\Gamma / 2\right) t / \hbar\right]$, where $\Gamma=\Gamma_{L}+\Gamma_{R}$. The exponential form of $G_{R}^{0}(t)$ allows us to evaluate $G(\tau, s, t)$ by a canonical transformation ${ }^{28}$ or by exponential resummation of low-order perturbation theory (Appendix B). We find

$$
G(\tau, s, t)=G_{R}^{0}(t)\left[G_{R}^{0}(s)\right]^{*} \exp \left[\frac{i(t-s) \lambda}{\hbar}-\sum_{\mathrm{q}}\left|\frac{M_{\mathrm{q}}}{\hbar \omega_{\mathrm{q}}}\right|^{2}\left[\left(1+2 N_{\omega_{\mathrm{q}}}\right) \operatorname{Re}\left(f_{\mathrm{q}}\right)+i \operatorname{Im}\left(f_{\mathrm{q}}\right)\right]\right),
$$

with

$$
f_{\mathbf{q}}(\tau, s, t)=2-e^{-i \omega_{\mathbf{q}} t}-e^{i \omega_{\mathbf{q}} s}+e^{-i \omega_{\mathbf{q}} \tau}\left(1-e^{i \omega_{\mathbf{q}} t}\right)\left(1-e^{i \omega_{\mathbf{q}} s}\right),
$$

where $\lambda=\Sigma_{\mathrm{q}}\left(M_{\mathrm{q}}^{2} / \hbar \omega_{\mathrm{q}}\right)$, and $N_{\omega_{\mathrm{q}}}$ is the Bose-Einstein occupation factor for a phonon mode of energy $\hbar \omega_{\mathrm{q}}$.

Although direct evaluation of the transmission matrix $T\left(\varepsilon^{\prime}, \varepsilon\right)$ in Eq. (13) for the general phonon spectrum will require three numerical Fourier transforms, the evaluation of the total transmission probability $T_{\text {tot }}(\varepsilon)$ requires only a single Fourier transform. $T_{\text {tot }}(\varepsilon)$ is defined as the transmission probability of a particle incident with energy $\varepsilon$, regardless of its final-state energy,

$$
\begin{aligned}
T_{\text {tot }}(\varepsilon) & =\int d \varepsilon^{\prime} T\left(\varepsilon^{\prime}, \varepsilon\right) \\
& =\int d \varepsilon^{\prime} \Gamma_{L}(\varepsilon) \Gamma_{R}\left(\varepsilon^{\prime}\right) \iiint \frac{d \tau d s d t}{2 \pi \hbar^{3}} e^{\left[\left(\varepsilon-\varepsilon^{\prime}\right) \tau+\varepsilon^{\prime} t-\varepsilon s\right] / \hbar} G(\tau, s, t) .
\end{aligned}
$$


In the wide-band limit, the couplings to the contacts will be energy independent and the integral over final-state energies $\varepsilon^{\prime}$ can be performed first to give a $\delta$ function $\delta(t-\tau)$. Using the $\delta$ function to integrate over $\tau$, we find

$$
T_{\mathrm{tot}}(\varepsilon)=\Gamma_{L} \Gamma_{R} \iint \frac{d s d t}{\hbar^{2}} e^{i \varepsilon(t-s) / \hbar} G(t, s, t)
$$

It is possible to reduce expression $(21)$ for $T_{\text {tot }}(\varepsilon)$ to a single integral by changing to sum and difference variables $T=(t+s) / 2$ and $\sigma=t-s$ :

$T_{\text {tot }}(\varepsilon)=\Gamma_{L} \Gamma_{R} \int_{-\infty}^{\infty} \frac{d \sigma}{\hbar} \int_{|\sigma| / 2}^{\infty} \frac{d T}{\hbar} \exp \left[-\frac{\Gamma T}{\hbar}+\frac{i\left(\varepsilon-\varepsilon_{0}+\lambda\right) \sigma}{\hbar}-\sum_{\mathrm{q}}\left|\frac{M_{\mathrm{q}}}{\hbar \omega_{\mathrm{q}}}\right|^{2}\left\{\left(1+2 N_{\omega_{\mathrm{q}}}\right) \operatorname{Re}\left[f_{\mathrm{q}}(\sigma)\right]+i \operatorname{Im}\left[f_{\mathrm{q}}(\sigma)\right]\right\}\right]$,

and integrating over the sum variable $T$ gives

$T_{\text {tot }}(\varepsilon)=\frac{\Gamma_{L} \Gamma_{R}}{\Gamma} \int_{-\infty}^{\infty} \frac{d \sigma}{\hbar} \exp \left[-\frac{\Gamma|\sigma|}{2 \hbar}+\frac{i\left(\varepsilon-\varepsilon_{0}+\lambda\right) \sigma}{\hbar}-\sum_{\mathbf{q}}\left|\frac{M_{\mathrm{q}}}{\hbar \omega_{\mathrm{q}}}\right|^{2}\left\{\left(1+2 N_{\omega_{\mathrm{q}}}\right) \operatorname{Re}\left[f_{\mathrm{q}}(\sigma)\right]+i \operatorname{Im}\left[f_{\mathrm{q}}(\sigma)\right]\right\}\right)$,

where

$$
f_{\mathrm{q}}(\sigma)=1-e^{-i \omega_{\mathrm{q}} \sigma}
$$

Since in many cases of interest, e.g., calculation of a resonant-tunneling current, only the total transmission probability is required, it may be convenient to evaluate $T_{\text {tot }}(\varepsilon)$ directly from Eq. (23) rather than first calculating the full transmission matrix $T\left(\varepsilon^{\prime}, \varepsilon\right)$. From Eq. (23) it is straightforward to confirm that the noninteracting total transmission probability in the wide-band limit is a Lorentzian,

$$
T_{\text {tot }}^{0}(\varepsilon)=\frac{\Gamma_{L} \Gamma_{R}}{\left(\varepsilon-\varepsilon_{0}\right)^{2}+(\Gamma / 2)^{2}}
$$

as required for an isolated resonance. ${ }^{29}$
Sum rules. Certain sum rules and simple analytic results valid for all phonon spectra in the wide-band limit, i.e., where the energy dependence of $\Gamma$ can be neglected, are derivable from the general solution (19) for $G(\tau, s, t)$. First, integrating the total transmission probability $T_{\text {tot }}(\varepsilon)$ in Eq. (23) over incident energies were find

$$
\int d \varepsilon T_{\text {tot }}(\varepsilon)=2 \pi \Gamma_{L} \Gamma_{R} / \Gamma,
$$

independent of the electron-phonon interaction. Second, the center of the transmission resonance is fixed at

$$
\langle\varepsilon\rangle=\frac{\Gamma}{2 \pi \Gamma_{L} \Gamma_{R}} \int d \varepsilon \varepsilon T_{\mathrm{tot}}(\varepsilon)=\varepsilon_{0},
$$

also independent of the electron-phonon interaction. To prove this, rewrite $T_{\text {tot }}(\varepsilon)$ in terms of $T\left(\varepsilon^{\prime}, \varepsilon\right)$ from Eq. (13):

$$
\langle\varepsilon\rangle=\frac{\Gamma}{2 \pi \Gamma_{L} \Gamma_{R}} \int d \varepsilon^{\prime} \int d \varepsilon \varepsilon \Gamma_{L} \Gamma_{R} \iiint \frac{d \tau d s d t}{2 \pi \hbar^{3}} e^{i\left[\left(\varepsilon-\varepsilon^{\prime}\right) \tau+\varepsilon^{\prime} t-\varepsilon s\right] / \hbar} G(\tau, s, t)
$$

The factor of $\varepsilon$ can be turned into a time derivative by $\varepsilon e^{-i \varepsilon s / \hbar}=i \hbar(d / d s)\left(e^{-i \varepsilon s / \hbar}\right)$. Following integration by parts over $s$, the energy integrals can be performed to produce $\delta$ functions of the time arguments, yielding

$$
\langle\varepsilon\rangle=-\left.i \Gamma \int d t\left(\frac{d}{d s} G(t, s, t)\right)\right|_{s=t} .
$$

The derivative of $G(t, s, t)$ is straightforward to obtain from Eq. (19),

$$
\begin{aligned}
\left.\int \frac{d}{d s} G(t, s, t)\right]\left.\right|_{s=t} & \\
= & {\left[\Theta(t)\left(i \varepsilon_{0}-\Gamma / 2\right) / \hbar+\delta(t) / 2\right] e^{-\Gamma t / \hbar}, }
\end{aligned}
$$

so that by inspection $\langle\varepsilon\rangle=\varepsilon_{0}$. The same procedure applied to the second energy moment gives the phonon broadening of the transmission resonance,

$$
\begin{aligned}
\delta\left\langle\varepsilon^{2}\right\rangle & =\frac{\Gamma}{2 \pi \Gamma_{L} \Gamma_{R}} \int d \varepsilon \varepsilon^{2}\left[T_{\text {tot }}(\varepsilon)-T_{\text {tot }}^{0}(\varepsilon)\right] \\
& =\sum_{\mathbf{q}}\left|M_{\mathbf{q}}\right|^{2}\left(1+2 N_{\omega_{\mathbf{q}}}\right)
\end{aligned}
$$

a quantity which is evidently always positive. Although the second moment of $T_{\text {tot }}(\varepsilon)$ itself cannot be defined because the line shape decays as $1 / \varepsilon^{2}$ in the tails, a useful estimate of the phonon-induced broadening can still be obtained from Eq. (31) if the important contributions to $\delta\left\langle\varepsilon^{2}\right\rangle$ are from the region near the resonance. This condition is satisfied in the case of an Einstein band of phonons-which we analyze next-where $\delta\left\langle\varepsilon^{2}\right\rangle$ approaches its asymptotic value within a few phonon energies of the resonance center.

Einstein phonons. We have explicitly evaluated the transmission matrix $T\left(\varepsilon^{\prime}, \varepsilon\right)$ for the simple case of an Einstein band of phonons with energy $\hbar \omega_{0}$ at zero temperature (Appendix C), 
$T\left(\varepsilon^{\prime}, \varepsilon\right)=\Gamma_{L} \Gamma_{R} e^{-2 g} \sum_{m=0}^{\infty} \frac{g^{m}}{m !} \delta\left(\varepsilon-\varepsilon^{\prime}-m \hbar \omega_{0}\right)\left|\sum_{j=0}^{m}(-1)^{j}\left(\begin{array}{l}m \\ j\end{array}\right] \sum_{l=0}^{\infty} \frac{g^{l}}{l !}\left(\frac{1}{\left[\varepsilon-\left(\varepsilon_{0}-\lambda\right)-(j+l) \hbar \omega_{0}\right]+i \Gamma / 2}\right)\right|^{2}$,

where the coupling constant $g=\Sigma_{\mathrm{q}}\left|M_{\mathrm{q}} / \hbar \omega_{0}\right|^{2}$. In Fig. 2, we have plotted $T_{\text {tot }}(\varepsilon)$ for the Einstein model with coupling constants $g=0.1,0.2,0.5$, and 1.0 and with no electron-phonon coupling for comparison. The sum rules, Eqs. (26) and (27), imply that the integrals under all the curves are equal and that their centers are at the same energy. At zero temperature, no phonons are present and the phonon-assisted resonances appear only at energies above the elastic resonance. The centers of the transmission curves remain fixed, however, because of the overall shift downward in energy by $\lambda=g \hbar \omega_{0}$ associated with the deformation of the lattice about the tunneling electron. In Fig. 3 we have plotted the phonon-induced broadening,

$\delta\left\langle\varepsilon^{2}\right\rangle=\frac{\Gamma}{2 \pi \Gamma_{L} \Gamma_{R}} \int_{-\Delta \varepsilon}^{\Delta \varepsilon} d \varepsilon \varepsilon^{2}\left[T_{\text {tot }}(\varepsilon)-T_{\text {tot }}^{0}(\varepsilon)\right]$,

for the transmission spectra from Fig. 2 as functions of the cutoff $\Delta \varepsilon$ of the region of integration in $\varepsilon$. While the

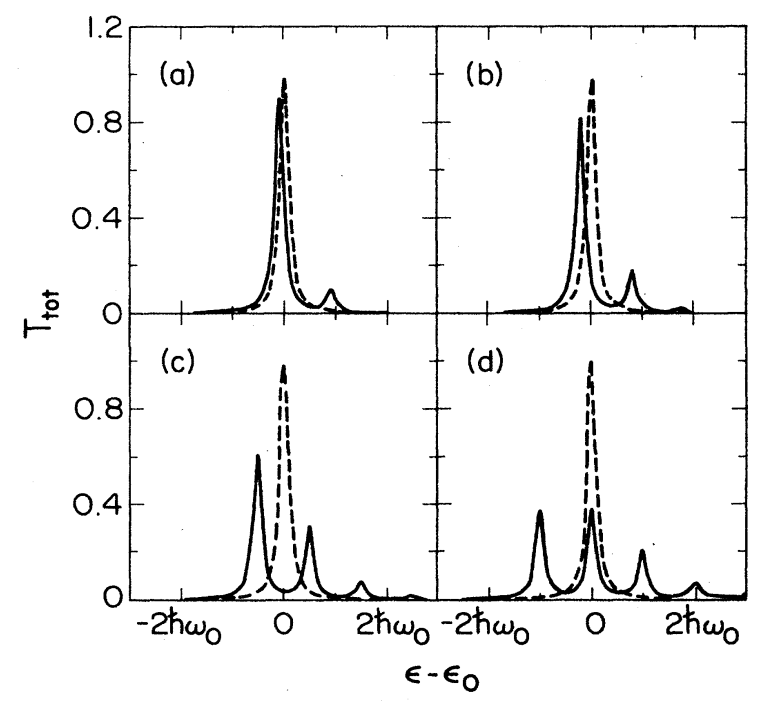

FIG. 2. Total transmission probability $T_{\text {tot }}(\varepsilon)$ vs incident energy for an electron interacting with Einstein phonons as it tunnels through a double-barrier structure, for four different coupling strengths: (a) $g=0.1$, (b) $g=0.2$, (c) $g=0.5$, and (d) $g=1.0$. In each case, the elastic resonance width is $\Gamma=0.2 \hbar \omega_{0}$ and the noninteracting probability is plotted as a dashed curve. If the couplings to the leads, $\Gamma_{L}$ and $\Gamma_{R}$, are not equal, then the transmission probability is reduced by a factor $4 \Gamma_{L} \Gamma_{R} / \Gamma$. The phonon-assisted transmission resonances lie one or more phonon energies above the main, elastic resonance. Because the lattice temperature is $T=0 \mathrm{~K}$, there are no resonances associated with phonon absorption. Sum rules guarantee that the integrated transmission probability and the center of the transmission curves in energy are independent of the electron-phonon interaction. transmission probabilities all have diverging second moments [recall that the noninteracting transmission spectrum $T_{\text {tot }}^{0}(\varepsilon)$ is a Lorentzian (25)], the broadening $\delta\left\langle\varepsilon^{2}\right\rangle$ is always well defined, and reaches its asymptotic value of $g\left(\hbar \omega_{0}\right)^{2}$ within a few phonon energies of the resonance center.

\section{ESCAPE RATE FROM THE QUANTUM WELL}

In addition to the sum rules governing $T_{\text {tot }}(\varepsilon)$, another model property that proves to be independent of the electron-phonon interaction in the wide-band limit is the escape rate of a particle from the resonant site. If an electron is placed on the site at $t_{0}=0$, then the thermally averaged probability of finding it still there at time $t>0$ is

$$
\begin{aligned}
P(t) & =\sum_{\alpha_{f}, \alpha_{i}} \frac{e^{-\beta E_{\alpha_{i}}}}{Z}\left|\left\langle 0, \alpha_{f}\left|c(t) c^{\dagger}(0)\right| 0, \alpha_{i}\right\rangle\right|^{2} \\
& =\left\langle c(0) c^{\dagger}(t) c(t) c^{\dagger}(0)\right\rangle .
\end{aligned}
$$

The expectation value for $P(t)$ in Eq. (34) is just a specific case of the Green function $G(\tau, s, t)$, namely $G(t, t, t)$. From Eq. (19) we can immediately determine the probability of remaining on the site since $f_{\mathrm{q}}(t, t, t)=0$,

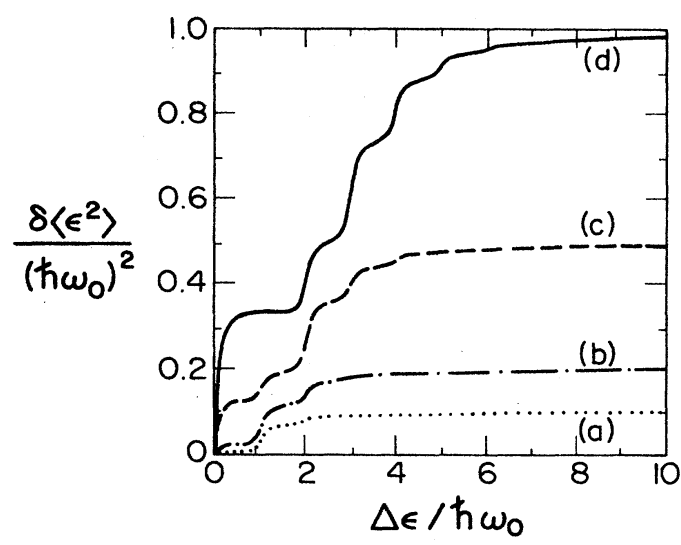

FIG. 3. Differences $\delta\left\langle\varepsilon^{2}\right\rangle$ between the normalized, second energy moments of the interacting and noninteracting transmission probabilities plotted as functions of the energy range $\Delta \varepsilon$ over which the differences are calculated, (33). The curves correspond to the different couplings used in Fig. 2: dotted, $g=0.1$; dashed-dotted, $g=0.2$; dashed, $g=0.5$; solid, $g=1.0$. In all cases, $\delta\left\langle\varepsilon^{2}\right\rangle$ approaches its asymptotic value $g\left(\hbar \omega_{0}\right)^{2}$ within a few phonon energies of the resonance center. Therefore, formula (31) for $\delta\left\langle\varepsilon^{2}\right\rangle$ provides a useful estimate of the phonon-induced broadening, even though the transmission probabilities decay as $1 / \varepsilon^{2}$ in the tails so that only the difference in second moments can be defined. 


$$
P(t)=G(t, t, t)=\left|G_{R}^{0}(t)\right|^{2}=e^{-\Gamma t / \hbar} .
$$

A particle placed on the resonant site escapes into the contacts with a decay lifetime $\hbar / \Gamma$, independent of the strength or spectrum of the phonon coupling.

By considering the elastic transmission amplitudes as analytic functions of energy, Price ${ }^{29}$ showed that each transmission resonance of a one-dimensional potential is associated with an exponentially decaying solution of the Schrödinger equation. The elastic escape lifetime $\tau_{\mathrm{el}}$, which is the inverse of the rate of decay, is therefore necessarily related to the elastic transmission width $\Gamma$ by

$$
\Gamma \tau_{\mathrm{el}} \simeq \hbar \text {. }
$$

The results of the preceding two sections demonstrate that this equality does not extend to the inelastic transmission width and escape lifetime in the presence of phonons. From Eq. (35) the inelastic lifetime and the elastic lifetime are equal, but the inelastic transmission width is always greater than the elastic width (31). In the case of an Einstein band, the interaction with phonons generates transmission sidebands each of which still has the elastic width $\Gamma$. Relation (36), therefore, applies to each sideband individually, but does not apply to the total transmission spectrum since its width is set by the envelope over the sidebands. In the case that the phonon frequencies are smaller than the elastic width so that the sidebands overlap, there will appear to be a single broad resonance; however, the resonance width which determines the characteristic decay time will continue to be that of the individual sidebands, i.e., $\Gamma$.

\section{THE RESONANT-TUNNELING CURRENT}

To predict the experimental effects of electron-phonon interaction in a resonant-tunneling structure it is necessary to calculate the current. Since our calculation of tunneling probabilities to this point has been restricted to one dimension, we will first evaluate the current for a quasi-one-dimensional resonant-tunneling structure. Efforts are presently underway ${ }^{30}$ to observe onedimensional resonant tunneling by transverse confinement of the electronic wave functions. Already, in the GaAs/ $/ \mathrm{Al}_{x} \mathrm{Ga}_{1-x} \mathrm{As}$ system, electrostatic confinement to approximately $600 \AA$ has been achieved. ${ }^{31}$ We calculate the one-dimensional current for the important case of polar-optic phonons using the result, Eq. (32), for the transmission probability $T_{\text {tot }}(\varepsilon)$ in the presence of an Einstein band of oscillators. The signature of optical-phonon interactions is found to be the appearance of phonon replicas of the main elastic peak in the valley region following the first transmission peak in the current-voltage characteristic. We begin by calculating the electronphonon coupling constant $g$ appropriate for polar-optical phonons in a quasi-one-dimensional structure.

The coupling of a localized electronic state to the three-dimensional phonons of the host material is well known in solid-state physics. ${ }^{32}$ Since the dominant electron-phonon coupling in $\mathrm{GaAs}$ and other polar semiconductors is to longitudinal-optical (LO) phonons, we focus on these modes. Because of their flat dispersion near the zone center, the LO phonons can be approximated by an Einstein band, for which the transmission matrix $T\left(\varepsilon^{\prime}, \varepsilon\right)$ is given in Eq. (32). It remains to determine the coupling constant

$$
g=\sum_{\mathbf{q}}\left|\frac{\boldsymbol{M}_{\mathbf{q}}}{\hbar \omega_{0}}\right|^{2}
$$

which appears in $T\left(\varepsilon^{\prime}, \varepsilon\right)$ in terms of the confinement size of the electronic state and known material parameters.

For the case of longitudinal-optical phonons of energy $\hbar \omega_{0}$, the electron-phonon matrix elements reduce to ${ }^{33}$

$$
M_{\mathrm{q}}=\frac{M \rho(\mathrm{q})}{q V^{1 / 2}},
$$

where $V$ is the volume, $\rho(q)$ is the qth component of the electron density, and the coupling strength $M$ can be expressed in terms of of the high- and low-frequency dielectric constants as

$$
M^{2}=2 \pi e^{2} \hbar \omega_{0}\left(\frac{1}{\epsilon_{\infty}}-\frac{1}{\epsilon_{0}}\right) .
$$

Since $g$ is sensitive only to the size of the electronic state, not to the detailed shape, it is convenient to assume a spherical wave function

$$
\phi(r)=\frac{\left(L_{0}\right)^{1 / 2}}{\pi} \frac{1}{r^{2}+\left(L_{0} / 2\right)^{2}},
$$

which has a characteristic full width $L_{0}$ at half maximum. The electron density at wave vector $q$ then has the simple form

$$
\rho(q)=e^{-q L_{0} / 2} .
$$

Consequently, we find the coupling of a localized state of width $L_{0}$ to longitudinal-optical phonons to be

$$
\left.g=\sum_{\mathbf{q}}\left|\frac{M_{\mathbf{q}}}{\hbar \omega_{0}}\right|^{2}=\frac{e^{2}}{\pi L_{0} \hbar \omega_{0}} \mid \frac{1}{\epsilon_{\infty}}-\frac{1}{\epsilon_{0}}\right)\left(1-e^{-q_{D} L_{0}}\right) .
$$

The correction $e^{-q_{D} L_{0}}$ comes from the cutoff of the phonon spectrum at the Debye wave vector $q_{D}$ and is negligible for a state which extends over many lattice constants.

For the III-V compound semiconductor GaAs, the relevant material parameters $\operatorname{are}^{34} \hbar \omega_{0}=36 \mathrm{meV}$, $\epsilon_{\infty}=10.9$, and $\epsilon_{0}=13.18$, leading to a coupling strength

$$
g_{\mathrm{GaAs}}=2.02 / L_{0}(\AA) \text {, }
$$

where $L_{0}$ is the full width in angstroms of the localized state. The coupling to optical phonons is stronger in the II-VI compounds. In the case of CdTe, the material parameters $\operatorname{are}^{35} \hbar \omega_{0}=21.2 \mathrm{meV}, \epsilon_{\infty}=7.21$, and $\epsilon_{0}=10.6$, leading to

$$
g_{\text {CdTe }}=9.59 / L_{0}(\AA),
$$

an enhancement of almost a factor of 5 over GaAs.

The total current $J$ through a quasi-one-dimensional resonant-tunneling structure in the absence of scattering 
can be expressed as a difference between right- and leftflowing currents. Specifically,

$$
\begin{aligned}
J_{0}= & (q / \pi) \int_{0}^{\infty} d k_{L} v_{k L} T_{\text {tot }}^{0}\left(\varepsilon_{k L}\right) f_{L}\left(\varepsilon_{k L}\right)\left[1-f_{R}\left(\varepsilon_{k L}\right)\right] \\
& -(q / \pi) \int_{0}^{\infty} d k_{R} v_{k R} T_{\text {tot }}^{0}\left(\varepsilon_{k R}\right) f_{R}\left(\varepsilon_{k R}\right)\left[1-f_{L}\left(\varepsilon_{k R}\right)\right],
\end{aligned}
$$

where $q$ is the carrier charge, $v_{k L}$ and $v_{k R}$ are velocities, $f_{L}(\varepsilon)$ and $f_{R}(\varepsilon)$ are the occupation functions in the left and right contacts, and $T_{\text {tot }}^{0}(\varepsilon)$ is the elastic transmission probability. By changing variables to energy, the density of states factors cancel the velocities, $v_{k}=(1 / \hbar) d \varepsilon_{k} / d k$, leaving

$$
J_{0}=(q / \pi \hbar) \int d \varepsilon T_{\text {tot }}^{0}(\varepsilon)\left[f_{L}(\varepsilon)-f_{R}(\varepsilon)\right] .
$$

The fact that the transmission probability at a given energy is the same for left- and right-going particles ${ }^{36}$ ensures that no net current will flow at equilibrium. Typically, the incident distributions will be of Fermi-Dirac type, in which case

$$
J_{0}=(q / \pi \hbar) \int d \varepsilon T_{\text {tot }}^{0}(\varepsilon)\left[f_{\mathrm{FD}}(\varepsilon)-f_{\mathrm{FD}}(\varepsilon+\Delta \mu)\right] \text {, }
$$

where $\Delta \mu$ is the difference in chemical potentials between the two leads, as indicated in Fig. 4. Energies are measured with respect to the conduction-band edge in the upstream lead so that $T_{\text {tot }}^{0}(\varepsilon)=0$ for $\varepsilon<0$.

In the interacting system, the expression for the current as a sum over independent channels is no longer exact. Since electrons can scatter during tunneling, both the exclusion principle and Coulomb shifts of the resonant-level energy influence the transmission probability. At the high biases typically required to bring the resonant level below the Fermi energy, however, both the backward current from the right lead and the exclusion from filled final states will be small effects, causing contributions to the current of order $e^{-\Delta \mu / k_{B} T}$. The current in the pres-

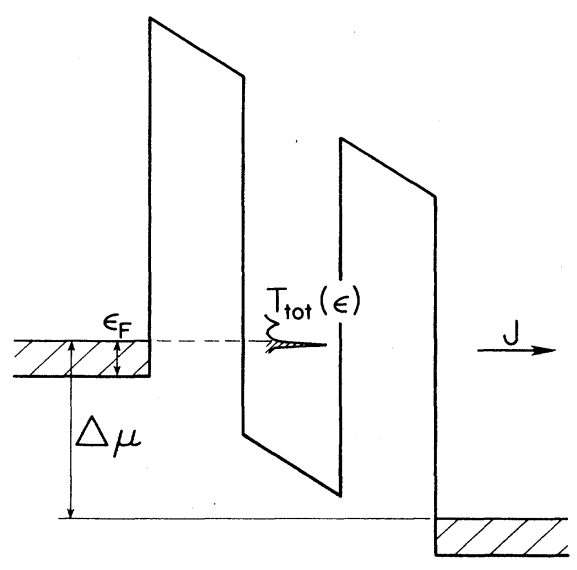

FIG. 4. Schematic drawing of a resonant-tunneling structure carrying a current $J$ between two doped contacts with Fermi energies $\varepsilon_{F}$. The current as a function of bias $\Delta \mu$ depends on the interacting transmission spectrum $T_{\text {tot }}(\varepsilon)$, an example of which is sketched in the well region. ence of electron-phonon interactions is therefore approximately given by

$$
J=(q / \pi \hbar) \int d \varepsilon T_{\mathrm{tot}}(\varepsilon) f_{\mathrm{FD}}(\varepsilon),
$$

where the elastic-transmission probability $T_{\text {tot }}^{0}(\varepsilon)$ has been replaced by the total transmission probability

$$
T_{\text {tot }}(\varepsilon)=\int d \varepsilon^{\prime} T\left(\varepsilon^{\prime}, \varepsilon\right),
$$

with $T_{\text {tot }}(\varepsilon)=0$ for $\varepsilon<0$. Under the assumption that the elastic couplings $\Gamma_{L}$ and $\Gamma_{R}$ can be taken constant, the exact solution for $T\left(\varepsilon^{\prime}, \varepsilon\right)$ obtained for an Einstein band of phonons (32) can be substituted into Eqs. (48) and (49) to obtain the current including the interaction of the tunneling electrons with optical phonons.

In Fig. 5, we have plotted the current $J$ in Eq. (48) for four different coupling constants, versus the energy of the resonant level. To make direct comparison to experiment, one must additionally relate the resonant-level energy to the total bias via a calculation of the selfconsistent potential. ${ }^{37}$ However, since the relation between resonant-level energy and bias is smooth, the shape and magnitude of the structure due to electron-phonon interaction will be the same in a current-voltage plot as in

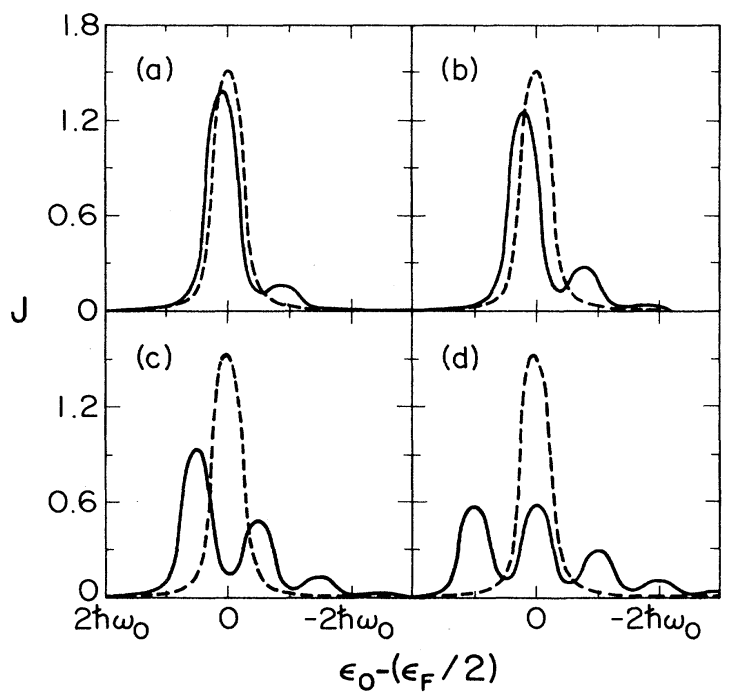

FIG. 5. Current vs resonant-level energy for a quasi-onedimensional resonant-tunneling structure, at four different coupling strengths: (a) $g=0.1$, (b) $g=0.2$, (c) $g=0.5$, and (d) $g=1.0$, calculated from Eq. (48). In each case, the Fermi energy is taken to be half the optical-phonon energy $\varepsilon_{F}=0.5 \hbar \omega_{0}$, the resonance width is $\Gamma=0.2 \hbar \omega_{0}$, and the dashed curve is for no electron-phonon interaction. The replica peaks to the right of the main current peak are due to phonon-emission-assisted resonant tunneling of electrons beginning one or more opticalphonon energies above the resonance. At $T=0 \mathrm{~K}$ there are no thermal phonons to give rise to phonon-absorption peaks. The first, Eq. (26), and second, Eq. (27), sum rules governing $T_{\text {tot }}(\varepsilon)$ ensure that the normalization of the current and its first energy moment $\left\langle\varepsilon_{0}\right\rangle=\varepsilon_{F} / 2$ are independent of the interaction with phonons. For convenience the current is plotted in units of $2 q \Gamma_{L} \Gamma_{R} \varepsilon_{F} / \hbar^{2} \omega_{0} \Gamma$ so that the integrals are normalized to 1 . 
a plot versus resonant-level energy. Even for the smallest coupling constant, the current has a phonon-assisted transmission peak at an energy for which there is insignificant elastic transmission. In a current-voltage plot, this peak would fall in the valley region between the first and second transmission resonances and could therefore be clearly resolved. Because the transmission probabilities $T_{\text {tot }}(\varepsilon)$ are evaluated at $T=0 \mathrm{~K}$ there are no peaks due to phonon absorption.

The sum rules in Eqs. (26), (27), and (31) governing the total transmission probability $T_{\text {tot }}(\varepsilon)$ have counterparts governing the current as a function of resonant-level energy. Because $T_{\text {tot }}(\varepsilon)$ in the wide-band limit depends only on the energy difference $\varepsilon-\varepsilon_{0}$, the integral over $\varepsilon$ at fixed $\varepsilon_{0}$ and the integral over $\varepsilon_{0}$ at fixed $\varepsilon$ are equal. Therefore, the following sum rules apply to the onedimensional current (48) at $T=0 \mathrm{~K}$ :

$$
\begin{aligned}
& \int d \varepsilon_{0} J\left(\varepsilon_{0}\right)=\frac{2 q \Gamma_{L} \Gamma_{R} \varepsilon_{F}}{\hbar \Gamma}, \\
& \left\langle\varepsilon_{0}\right\rangle=\frac{\int d \varepsilon_{0} \varepsilon_{0} J\left(\varepsilon_{0}\right)}{\int d \varepsilon_{0} J\left(\varepsilon_{0}\right)}=\frac{\varepsilon_{F}}{2}, \\
& \delta\left\langle\varepsilon_{0}^{2}\right\rangle=\frac{\int d \varepsilon_{0} \varepsilon_{0}^{2}\left[J\left(\varepsilon_{0}\right)-J_{0}\left(\varepsilon_{0}\right)\right]}{\int d \varepsilon_{0} J\left(\varepsilon_{0}\right)}=\sum_{\mathbf{q}}\left|M_{\mathbf{q}}\right|^{2} .
\end{aligned}
$$

It is to illustrate these sum rules that the currents in Fig. 5 are plotted in units of $2 q \Gamma_{L} \Gamma_{R} \varepsilon_{F} / \hbar^{2} \omega_{0} \Gamma$, giving an overall normalization of 1 , and are centered at $\left\langle\varepsilon_{0}\right\rangle=\varepsilon_{F} / 2$.

In Fig. 6, we have plotted the current for a coupling

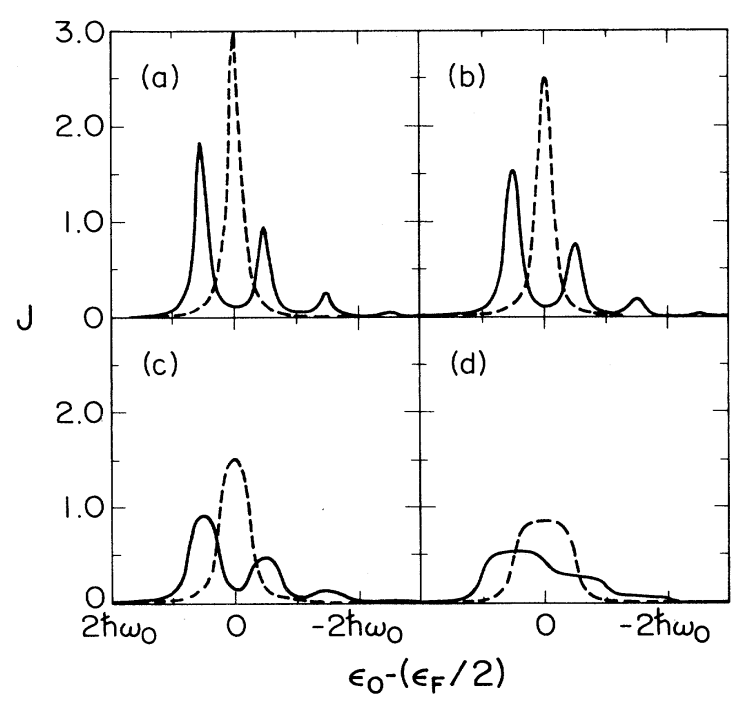

FIG. 6. Current vs resonant-level energy as in Fig. 5, for a coupling $g=0.5$ at four different Fermi energies: (a) $\varepsilon_{F}=0.1 \hbar \omega_{0}$, (b) $\varepsilon_{F}=0.2 \hbar \omega_{0}$, (c) $\varepsilon_{F}=0.5 \hbar \omega_{0}$, and (d) $\varepsilon_{F}=1.0 \hbar \omega_{0}$, calculated from Eq. (48). The dashed curves are for no electron-phonon interaction. The units of current are $2 q \Gamma_{L} \Gamma_{R} \varepsilon_{F} / \hbar^{2} \omega_{0} \Gamma$ so that the integrals are normalized to 1 . Increasing the Fermi energy leads to an increase in the real current, but blurs the separation between phonon replica peaks. $g=0.5$ over a range of Fermi energies. Because of the factor of $\varepsilon_{F}$ in the normalization, the true current increases with Fermi energy; however, the resolution of the peaks is diminished. The width of each peak is roughly $\varepsilon_{F}$, the separation between peaks is $\hbar \omega_{0}$, and the falloff of each peak occurs over an energy $\Gamma$. The peaks can therefore only be resolved as long as the Fermi energy is small compared to the phonon energy. In typical structures the elastic width $\Gamma$ is much smaller than either $\varepsilon_{F}$ or $\hbar \omega_{0}$.

\section{THREE DIMENSIONS AND TRANSVERSE SCATTERING}

The results in the previous section for the onedimensional resonant-tunneling current are predictions of what will be observed in structures with strong transverse confinement of the electron wave function. The body of existing measurements, however, has been performed on structures with no confinement in the transverse direction. While it is straightforward to derive an expression for the transmission matrix for three-dimensional structures, it is no longer possible to evaluate the transport Green function $G(\tau, s, t)$ exactly, even in the wide-band limit. There are two ways to confront this difficulty: (1) In order to treat one-phonon, or two-phonon, processes the perturbative expansion in Appendix B can be generalized to three dimensions, or (2) if the transverse scattering is confined to small wave-vector changes, the change in the transverse part of the energy of the tunneling particle can be neglected, and an exact solution again exists in the wide-band limit. In this section we will present the formulas obtained from a perturbative expansion in the electron-phonon coupling strength and the solution for the wide-band limit obtained by neglecting changes in transverse momentum.

In three dimensions, the electronic Hamiltonian for resonant tunneling is

$$
\begin{gathered}
H_{e}=\sum_{\mathbf{k}_{t}} \varepsilon_{\mathbf{k}_{t}} c_{\mathbf{k}_{t}}^{\dagger} c_{\mathbf{k}_{t}}+\sum_{\mathbf{k}}\left(\varepsilon_{\mathrm{k} L} c_{\mathbf{k} L}^{\dagger} c_{\mathrm{k} L}+\varepsilon_{\mathrm{k} R} c_{\mathrm{k} R}^{\dagger} c_{\mathrm{k} R}\right) \\
+\sum_{\mathbf{k}}\left[V_{\mathrm{k} L}\left(c_{\mathrm{k} L}^{\dagger} c_{\mathbf{k}_{t}}+c_{\mathbf{k}_{t}}^{\dagger} c_{\mathrm{k} L}\right)\right. \\
\left.+V_{\mathrm{k} R}\left(c_{\mathrm{k} R}^{\dagger} c_{\mathbf{k}_{t}}+c_{\mathbf{k}_{t}}^{\dagger} c_{\mathrm{k} R}\right)\right]
\end{gathered}
$$

which differs from the one-dimensional Hamiltonian only in the addition of a transverse degree of freedom labeled by momentum $\mathbf{k}_{t}$. Tunneling is assumed to conserve $\mathbf{k}_{t}$ so that the hopping matrix elements $V_{\mathrm{k} L}$ and $V_{\mathrm{k} R}$ connect only states with the same transverse momentum. The phonon Hamiltonian is unchanged from the onedimensional form,

$$
H_{\mathrm{ph}}=\sum_{\mathrm{q}} \hbar \omega_{\mathrm{q}} a_{\mathrm{q}}^{\dagger} a_{\mathrm{q}},
$$

since the phonons were always taken to be three dimensional. The electron-phonon interaction Hamiltonian is

$$
H_{\text {int }}=\sum_{\mathbf{q}} M_{\mathrm{q}}\left(a_{\mathrm{q}}+a_{-\mathrm{q}}^{\dagger}\right) \sum_{\mathbf{k}_{t}} c_{\mathbf{k}_{t}+\mathbf{q}_{t}}^{\dagger} c_{\mathbf{k}_{t}},
$$

which allows the tunneling electron to exchange trans- 
verse momentum as well as energy with the phonons, but explicitly conserves the total transverse momentum of the system.

In order to describe tunneling through a threedimensional structure, the transmission matrix $T\left(\varepsilon^{\prime}, \varepsilon\right)$ must be generalized to include the transverse degrees of freedom. We define a new transmission matrix $T\left(\mathbf{k}^{\prime}, \mathbf{k}\right)$ to be the probability, per unit final longitudinal momentum $k_{l}^{\prime}$, that a particle incident with momentum $\mathbf{k}$ will be transmitted with momentum $\mathbf{k}^{\prime}$. It is convenient to treat the transverse momenta as discrete quantum numbers while allowing the longitudinal momenta to form a continuum; this facilitates comparison with the onedimensional case in the limit of a single transverse state. The total transmission probability for an electron incident with momentum $\mathbf{k}$ will therefore be

$$
T_{\mathrm{tot}}(\mathbf{k})=\sum_{\mathbf{k}_{t}^{\prime}} \int d k_{l}^{\prime} T\left(\mathbf{k}^{\prime}, \mathbf{k}\right)
$$

The $S$-matrix derivation of the transmission matrix (Appendix A) is unchanged by the addition of a transverse degree of freedom; the result is

$$
\begin{aligned}
T\left(\mathbf{k}^{\prime}, \mathbf{k}\right)= & \Gamma_{L}(\mathbf{k}) \Gamma_{R}\left(\mathbf{k}^{\prime}\right)\left|\frac{d \varepsilon_{\mathbf{k}^{\prime} R}}{d k_{l}^{\prime}}\right| \\
& \times \iiint \frac{d \tau d s d t}{2 \pi \hbar^{3}} e^{i\left[\left(\varepsilon-\varepsilon^{\prime}\right) \tau+\varepsilon^{\prime} t-\varepsilon s\right] / \hbar} \\
& \times G_{\mathbf{k}_{t}^{\prime} \mathbf{k}_{t}}(\tau, s, t)
\end{aligned}
$$

where $\varepsilon=\varepsilon_{\mathrm{k} L}$ and $\varepsilon^{\prime}=\varepsilon_{\mathrm{k}^{\prime} R}$ are measured with respect to the same zero, the elastic resonance widths are

$\Gamma_{L(R)}(\mathbf{k})=2 \pi \sum_{\mathbf{k}^{\prime}}\left|V_{\mathbf{k}^{\prime} L\left(\mathbf{k}^{\prime} R\right)}\right|^{2} \delta_{\mathbf{k}_{t}^{\prime}, \mathbf{k}_{t}} \delta\left(\varepsilon_{\mathbf{k}}-\varepsilon_{\mathbf{k}^{\prime} L\left(\mathbf{k}^{\prime} R\right)}\right)$,

and the interacting Green function is

$G_{\mathbf{k}_{t}^{\prime} \mathbf{k}_{t}}(\tau, s, t)=\Theta(s) \Theta(t)\left\langle c_{\mathbf{k}_{t}}(\tau-s) c_{\mathbf{k}_{t}^{\prime}}^{\dagger}(\tau) c_{\mathbf{k}_{t}^{\prime}}(t) c_{\mathbf{k}_{t}}^{\dagger}(0)\right\rangle$.

The only changes from the one-dimensional result, Eqs. (13) and (14), are the additional transverse-momentum labels and the density-of-states factor $\left|d \varepsilon_{\mathrm{k}^{\prime} R} / d k_{l}^{\prime}\right|$ which appears because $T\left(\mathbf{k}^{\prime}, \mathbf{k}\right)$ gives the transmission probability per unit final longitudinal momentum instead of per unit final energy.

The leading terms in a perturbative expansion of $G_{\mathbf{k}_{t}^{\prime} \mathbf{k}_{t}}(\tau, s, t)$ correspond to the four diagrams appearing in

Fig. 7, which differ from their one-dimensional counterparts in Fig. 9 (in Appendix B) only by the inclusion of transverse-momentum labels. In the absence of electronphonon interaction, the two-particle Green function factors into the product of a one-particle Green function
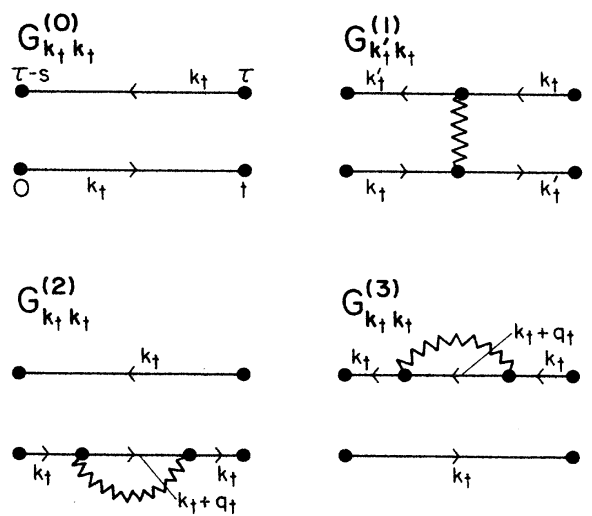

FIG. 7. Diagrammatic representation of the leading terms in a perturbative expansion of the interacting Green function $G_{\mathbf{k}_{t}^{\prime} \mathbf{k}_{t}}(\tau, s, t)$ for three-dimensional resonant tunneling. The diagrams differ from those appearing in the one-dimensional problem (cf. Fig. 9 in Appendix B) only in the presence of transverse-momentum labels. As in the one-dimensional case, the first diagram $G_{\mathbf{k}_{t}^{\prime} \mathbf{k}_{t}}^{(0)}$ consists of an electron Green function and its complex conjugate and is the sole term in the absence of electron-phonon interaction. The second diagram $G_{\mathbf{k}_{t}^{\prime} \mathbf{k}_{t}}^{(1)}$ includes a phonon line connecting the two electron lines and represents the emission or absorption of one real phonon. The last two diagrams $G_{\mathbf{k}_{t}^{\prime} \mathbf{k}_{t}}^{(2)}$ and $G_{\mathbf{k}_{t}^{\prime} \mathbf{k}_{t}}^{(3)}$ describe the effects of dressing of the electron lines by a virtual phonon.

and its complex conjugate, represented by the first diagram,

$$
G_{\mathbf{k}_{t}^{\prime} \mathbf{k}_{t}}^{(0)}(\tau, s, t)=\delta_{\mathbf{k}_{t}^{\prime}, \mathbf{k}_{t}} G_{\mathbf{k}_{t}}^{0}(t)\left[G_{\mathbf{k}_{t}}^{0}(s)\right]^{*},
$$

where the noninteracting one-particle Green function is defined by

$$
G_{\mathbf{k}_{t}}^{0}(t)=-i \Theta(t)\left\langle 0\left|c_{\mathbf{k}_{t}}(t) c_{\mathbf{k}_{t}}^{\dagger}(0)\right| 0\right\rangle,
$$

with

$$
c_{\mathrm{k}_{t}}(t)=e^{i H_{e} t / \hbar} c_{\mathrm{k}_{t}}(0) e^{-i H_{e} t / \hbar}
$$

The second diagram corresponds to emission or absorption of a single phonon. Since each phonon line between times $t_{1}$ and $t_{2}$ contributes a factor of

$$
\sum_{\mathbf{q}}\left|M_{\mathbf{q}}\right|^{2}\left[N_{\omega_{\mathbf{q}}} e^{i \omega_{\mathbf{q}}\left(t_{2}-t_{1}\right)}+\left(1+N_{\omega_{\mathbf{q}}}\right) e^{-i \omega_{\mathbf{q}}\left(t_{2}-t_{1}\right)}\right]
$$

where the sum is subject to conservation of transverse momentum and where $N_{\omega_{\mathrm{q}}}$ is the Bose-Einstein occupation factor for phonon mode $q$, the second diagram represents 


$$
\begin{aligned}
& G_{\mathbf{k}_{t}^{\prime} \mathbf{k}_{t}}^{(1)}(\tau, s, t)=\sum_{\mathbf{q}} \delta_{\mathbf{k}_{t}^{\prime}, \mathbf{k}_{t}+\mathbf{q}_{t}}\left|M_{\mathbf{q}}\right|^{2} \\
& \times\left[N _ { \omega _ { \mathbf { q } } } e ^ { i \omega _ { \mathbf { q } } \tau } \int _ { - \infty } ^ { \infty } d s ^ { \prime } \left[G_{\mathbf{k}_{t}^{\prime}}^{0}\left(s-s^{\prime}\right]^{*}\left[G_{\mathbf{k}_{t}}^{0}\left(s^{\prime}\right)\right]^{*} e^{-i \omega_{\mathbf{q}}\left(s-s^{\prime}\right)} \int_{-\infty}^{\infty} d t^{\prime} G_{\mathbf{k}_{t}^{\prime}}^{0}\left(t-t^{\prime}\right) G_{\mathbf{k}_{t}}^{0}\left(t^{\prime}\right) e^{-i \omega_{\mathbf{q}} t^{\prime}}\right.\right. \\
& +\left(1+N_{\omega_{\mathbf{q}}}\right) e^{-i \omega_{\mathbf{q}} \tau} \int_{-\infty}^{\infty} d s^{\prime}\left[G_{\mathbf{k}_{t}^{\prime}}^{0}\left(s-s^{\prime}\right)\right]^{*}\left[G_{\mathbf{k}_{t}}^{0}\left(s^{\prime}\right)\right]^{*} e^{i \omega_{\mathbf{q}}\left(s-s^{\prime}\right)} \\
& \left.\times \int_{-\infty}^{\infty} d t^{\prime} G_{\mathbf{k}_{t}^{\prime}}^{0}\left(t-t^{\prime}\right) G_{\mathbf{k}_{t}}^{0}\left(t^{\prime}\right) e^{i \omega_{\mathrm{q}} t^{\prime}}\right]
\end{aligned}
$$

as in Eq. (B1). Similarly, as in Eq. (B2), the effect of renormalization of the electron propagation due to virtual-phonon processes is represented by the third diagram,

$$
\begin{array}{r}
G_{\mathbf{k}_{t}^{\prime} \mathbf{k}_{t}}^{(2)}(\tau, s, t)=\delta_{\mathbf{k}_{t}^{\prime}, \mathbf{k}_{t}}\left[G_{\mathbf{k}_{t}}^{0}(s)\right]^{*} \sum_{\mathbf{q}}\left|M_{\mathbf{q}}\right|^{2} \int_{-\infty}^{\infty} d t_{2}^{\prime} \int_{-\infty}^{\infty} d t_{1}^{\prime} G_{\mathbf{k}_{t}}^{0}\left(t-t_{2}^{\prime}\right) G_{\mathbf{k}_{t}+\mathbf{q}_{t}}\left(t_{2}^{\prime}-t_{1}^{\prime}\right) G_{\mathbf{k}_{t}}^{0}\left(t_{1}^{\prime}\right) \\
\times\left[N_{\omega_{\mathrm{q}}} e^{i \omega_{\mathrm{q}}\left(t_{2}^{\prime}-t_{1}^{\prime}\right)}+\left(1+N_{\omega_{\mathrm{q}}}\right) e^{-i \omega_{\mathrm{q}}\left(t_{2}^{\prime}-t_{1}^{\prime}\right)}\right]
\end{array}
$$

Finally, from the symmetry between the last two terms in the perturbative expansion one finds

$$
G_{\mathbf{k}_{t}^{\prime} \mathbf{k}_{t}}^{(3)}(\tau, s, t)=\left[G_{\mathbf{k}_{t}^{\prime} \mathbf{k}_{t}}^{(2)}(\tau, t, s)\right]^{*} \text {. }
$$

The formulas in Eqs. (60) and (63)-(65) provide a starting point for the quantitative modeling of one-phonon processes in existing three-dimensional resonant-tunneling structures.

If one is interested in high-order electron-phonon processes, the perturbative approach becomes rapidly impractical. An alternative scheme is to neglect changes in the energy of transverse motion caused by scattering, which is reasonable if the interactions involve only small momentum transfers - as is the case for polar-optical phonons which couple most strongly near zone center (38). Within this approximation, the transverse momentum factors out of the transmission probability and the one-dimensional result applies to each transverse channel independently. The exact solution obtained in the wideband limit in one dimension can consequently be employed to treat multiple-phonon processes in three dimensions.

In the wide-band limit, the approximation that changes in transverse momentum are negligible is equivalent to replacing all the single-particle Green functions in Eqs. (60), (63), (64), and (65) by

$$
G_{R}^{0}(t)=-i \Theta(t) e^{\left(-i \varepsilon_{\mathrm{k}_{t}}-\Gamma / 2\right) t / \hbar},
$$

where $\mathbf{k}_{t}$ is the initial transverse momentum. The problem is therefore reduced to the one-dimensional case so exponential resummation of the leading diagrams is exact (Appendix B), and one finds

$$
T\left(k_{l}^{\prime}, k_{l}\right)=\sum_{\mathbf{k}_{t}^{\prime}} T\left(\mathbf{k}^{\prime}, \mathbf{k}\right)=\left|\frac{d \varepsilon_{\mathbf{k}^{\prime} R}}{d k_{l}^{\prime}}\right| T\left(\varepsilon_{l}^{\prime}, \varepsilon_{l}\right),
$$

where $\varepsilon_{l}=\varepsilon-\hbar^{2} k_{t}^{2} / 2 m *$ and $\varepsilon_{l}^{\prime}=\varepsilon^{\prime}-\hbar^{2} k_{t}^{\prime 2} / 2 m^{*}$ so the energy associated with transverse motion does not appear in the transmission matrix.

\section{THE CURRENT IN THREE DIMENSIONS}

In this section, we will calculate the phonon effects on the current in a three-dimensional resonant-tunneling structure. Since the dominant electron-phonon interaction in a polar semiconductor such as GaAs is with the longitudinal-optical phonons near zone center, we will use Eq. (67) to estimate the three-dimensional transmission matrix $T\left(\mathbf{k}^{\prime}, \mathbf{k}\right)$ in terms of the one-dimensional transmission matrix $T\left(\varepsilon_{l}^{\prime}, \varepsilon_{l}\right)$ for Einstein phonons, (32). As in the one-dimensional case, in order to calculate the current $J$ we assume that the total current at high bias can be written as a sum over momentum channels incident from the left lead,

$$
J=\frac{A q}{8 \pi^{3}} \int_{0}^{\infty} d k_{l} \int d^{2} k_{t} v_{l} f_{L}\left(\varepsilon_{\mathbf{k}}\right) T_{\text {tot }}(\mathbf{k}),
$$

where $A$ is the cross-sectional area, $v_{l}$ is the longitudinal velocity, and the total transmission probability is

$$
T_{\mathrm{tot}}(\mathbf{k})=\sum_{\mathbf{k}_{t}^{\prime}} \int d k_{l}^{\prime} T\left(\mathbf{k}^{\prime}, \mathbf{k}\right)=\int d \varepsilon_{l}^{\prime} T\left(\varepsilon_{l}^{\prime}, \varepsilon_{l}\right) .
$$

At low temperature, the integral over transverse momenta gives a factor of $4 \pi m^{*}\left(\varepsilon_{F}-\varepsilon_{l}\right) / \hbar^{2}$. Changing the final integral over longitudinal momentum to an integral over energy yields

$$
J=\frac{A q m^{*}}{2 \pi^{2} \hbar^{3}} \int_{0}^{\varepsilon_{F}} d \varepsilon_{l}\left(\varepsilon_{F}-\varepsilon_{l}\right) T_{\text {tot }}\left(\varepsilon_{l}\right)
$$

where $T_{\text {tot }}\left(\varepsilon_{l}\right)=T_{\text {tot }}(\mathbf{k})$ is the total interacting transmission probability found from Eq. (32).

The sum rules on the total transmission probability in Eqs. (26), (27), and (31) give rise to sum rules governing the three-dimensional current $J$ in Eq. (70) as a function of resonant-level energy. Specifically, at zero temperature we find,

$$
\begin{gathered}
\int d \varepsilon_{0} J\left(\varepsilon_{0}\right)=\frac{A q m^{*} \varepsilon_{F}^{2} \Gamma_{L} \Gamma_{R}}{2 \pi \hbar^{3} \Gamma}, \\
\left\langle\varepsilon_{0}\right\rangle=\frac{\int d \varepsilon_{0} \varepsilon_{0} J\left(\varepsilon_{0}\right)}{\int d \varepsilon_{0} J\left(\varepsilon_{0}\right)}=\varepsilon_{F} / 3,
\end{gathered}
$$




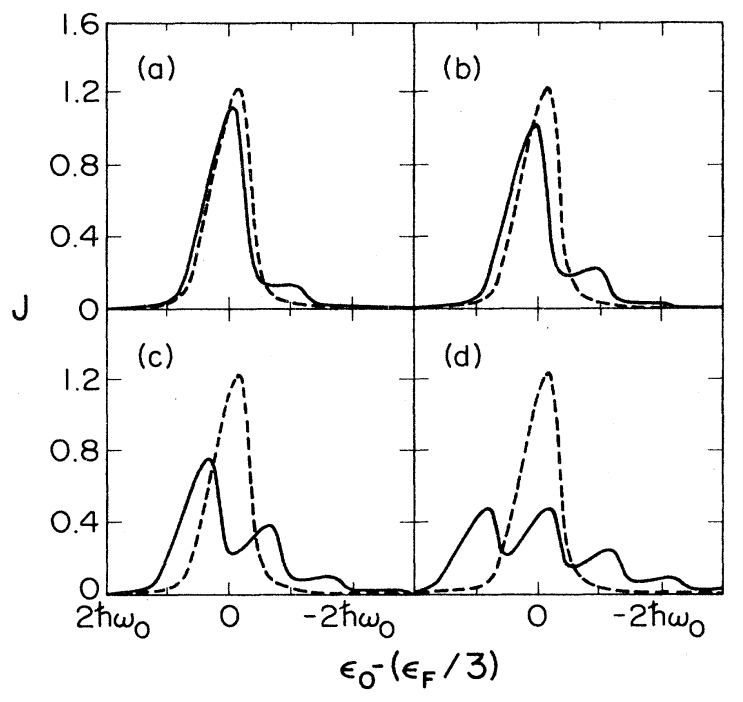

FIG. 8. Current vs resonant-level energy for a threedimensional resonant-tunneling structure at four different coupling strengths: (a) $g=0.1$, (b) $g=0.2$, (c) $g=0.5$, and (d) $g=1.0$, calculated from Eq. (70). For comparison, the dashed curves are for no coupling to phonons. In each case, the Fermi energy is taken to equal the optical-phonon energy $\varepsilon_{F}=\hbar \omega_{0}$ and the resonance width is $\Gamma=0.2 \hbar \omega_{0}$. Because of the sum rules governing the current given by Eqs. (71) and (72), the integrated weight under the curves and their average positions $\left\langle\varepsilon_{0}\right\rangle=\varepsilon_{F} / 3$ are independent of the interaction with phonons. The threedimensional current is plotted in units of $\operatorname{Aqm}^{*} \Gamma_{L} \Gamma_{R} \varepsilon_{F}^{2} /$ $2 \pi \hbar^{4} \omega_{0} \Gamma$ so that the integrals are normalized to 1 .

$$
\delta\left\langle\varepsilon_{0}^{2}\right\rangle=\frac{\int d \varepsilon_{0} \varepsilon_{0}^{2}\left[J\left(\varepsilon_{0}\right)-J_{0}\left(\varepsilon_{0}\right)\right]}{\int d \varepsilon_{0} J\left(\varepsilon_{0}\right)}=\sum_{\mathbf{q}}\left|M_{\mathbf{q}}\right|^{2} .
$$

In Fig. 8, we have plotted the current $J$ versus resonant-level energy for four values of the coupling constant $g$. The elastic resonance width is taken to be $\Gamma=0.2 \hbar \omega_{0}$ and the Fermi energy to be $\varepsilon_{F}=\hbar \omega_{0}$. The currents are plotted in units of $A q m^{*} \Gamma_{L} \Gamma_{R} \varepsilon_{F}^{2} / 2 \pi \hbar^{4} \omega_{0} \Gamma$, giving an overall normalization of 1 , and are centered at $\left\langle\varepsilon_{0}\right\rangle=\varepsilon_{F} / 3$ to illustrate the sum rules. The successive peaks in the current correspond to the one-phonon, twophonon, and three-phonon emission replicas of the main elastic peak. While the single-phonon peak has been seen experimentally by several groups, ${ }^{38,39}$ to our knowledge there has as yet been no observation of multiphonon processes in resonant tunneling.

\section{CONCLUSION}

In summary, we have applied scattering theory to obtain an expression (13) for the resonant-tunneling transmission probability $T\left(\varepsilon^{\prime}, \varepsilon\right)$ in the presence of inelastic scattering. In the wide-band limit, in which the density of states in the leads is constant and the tunneling matrix elements are independent of energy, an exact solution was obtained for $T\left(\varepsilon^{\prime}, \varepsilon\right)$ valid for any phonon spectrum, coupling strength, and temperature (19). Several sum rules in the wide-band limit were identified from the exact solution; in particular, the energy integral of the total transmission probability $T_{\text {tot }}(\varepsilon)$ and the first moment of $T_{\text {tot }}(\varepsilon)$ were found to be independent of the interaction with phonons. Also found to be independent of the phonons in the wide-band limit was the escape rate of an electron from the resonant site. Using the results for the transmission matrix $T\left(\varepsilon^{\prime}, \varepsilon\right)$ in the presence of an Einstein band of phonons (32), we calculated the resonanttunneling current expected for a quasi-one-dimensional structure including the interaction with polar-optical phonons. To facilitate the eventual comparison to experiment, we extended the scattering theory for transmission to three dimensions and again calculated the current in the presence of polar-optical phonons. At present, in experimental $\mathrm{GaAs} / \mathrm{Al}_{x} \mathrm{Ga}_{1-x} \mathrm{As}$ resonant-tunneling structures the couplings to phonons is typically weak enough that the lowest-order perturbative results found in Eqs. (60) and (63)-(65) are sufficient for purposes of modeling.

What remains unsolved is the problem of strong coupling to phonons when the electronic Hamiltonian is more complicated than a single level coupled to two wide bands. We hope that some of the methods we have developed may prove useful in the study of more realistic structures than those represented by the single-site model. For example, it is clear from the derivation in Appendix A that transmission through a series of quantum wells or through one quantum well with multiple levels can also be expressed in terms of a two-particle Green function. Our belief is that an exact formulation of transmission probabilities in terms of two-particle Green functions similar to $G(\tau, s, t)$ is possible for arbitrarily complicated structures connected to ideal leads.

\section{ACKNOWLEDGMENTS}

This work was supported by the U.S. Office of Naval Research. In addition, one of us (N.S.W.) gratefully acknowledges the support of the Fannie and John Hertz Foundation (Livermore, CA).

\section{APPENDIX A: REDUCING $T\left(\varepsilon^{\prime}, \varepsilon\right)$ TO A GREEN FUNCTION VIA THE $S$ MATRIX}

We apply the $S$-matrix formalism to find a practical expression (13) for the transmission matrix $T\left(\varepsilon^{\prime}, \varepsilon\right)$ introduced in Sec. II. Since the single-site model for resonant tunneling is already in the form of a scattering problem - free particles incident on a localized region of scattering - the $S$-matrix ${ }^{24}$ theory can be applied directly. The result is an exact formula for the transmission matrix in terms of a Green function of the resonant site. Existing techniques can then be applied to evaluate the Green function.

Scattering theory dictates that the Hamiltonian first be divided into bare and interaction parts, $H_{0}+H_{1}$. The Hamiltonian for resonant tunneling is accordingly divided into a noninteracting part, 


$$
\begin{aligned}
H_{0}= & \varepsilon_{0} c^{\dagger} c+\sum_{k} \varepsilon_{\mathrm{k} L} c_{\mathrm{k} L}^{\dagger} c_{\mathbf{k} L} \\
& +\sum_{k} \varepsilon_{\mathbf{k} R} c_{\mathbf{k} R}^{\dagger} c_{\mathbf{k} R}+\sum_{\mathbf{q}} \hbar \omega_{\mathrm{q}} a_{\mathrm{q}}^{\dagger} a_{\mathbf{q}},
\end{aligned}
$$

and an interaction part that contains both the electronphonon interaction and the electron tunneling between the resonant site and the contacts,

$$
\begin{aligned}
H_{1}= & c^{\dagger} c \sum_{\mathbf{q}} M_{\mathbf{q}}\left(a_{\mathbf{q}}^{\dagger}+a_{-\mathrm{q}}\right)+\sum_{k} V_{\mathrm{k} L}\left(c_{\mathrm{k} L}^{\dagger} c+c^{\dagger} c_{\mathrm{k} L}\right) \\
& +\sum_{k} V_{\mathbf{k} R}\left(c_{\mathrm{k} R}^{\dagger} c+c^{\dagger} c_{\mathrm{k} R}\right)
\end{aligned}
$$

The noninteracting electron eigenstates are standing waves confined in either the left or right contact plus a single localized state, e.g., an impurity level or the ground state of a quantum well. For convenience, we will label the basis states by energy instead of wave vector; in our notation $|\varepsilon, \alpha, L(R)\rangle$ denotes an electron of energy $\varepsilon$ in the left (right) contact with the phonon system in state $\alpha$. States in different contacts are orthogonal since they have no spatial overlap, while states in the same contact, say the left, are normalized according to

$$
\left\langle\varepsilon^{\prime}, \alpha^{\prime}, L \mid \varepsilon, \alpha, L\right\rangle=\delta_{\alpha^{\prime}, \alpha} \delta\left(\varepsilon^{\prime}-\varepsilon\right) .
$$

The second step in the application of the $S$ matrix is to form an initial incoming wave packet from the noninteracting states in the left contact,

$$
\left|\phi_{\varepsilon_{i}}, \alpha_{i}, L\right\rangle=\int d \varepsilon \frac{1}{\sqrt{\Delta \varepsilon}} \phi\left[\frac{\varepsilon-\varepsilon_{i}}{\Delta \varepsilon}\right]\left|\varepsilon, \alpha_{i}, L\right\rangle,
$$

where the wave packet is normalized to represent a single particle,

$$
\int d u|\phi(u)|^{2}=1 \text {. }
$$

The energy width $\Delta \varepsilon$ of the incident wave packet is initially assumed finite to allow the particle to be localized far from the interaction region. Eventually, the energy width will be taken to zero, after the final state of the wave packet has been determined.

The $S$ matrix acts on an initial incoming wave packet to produce the final outgoing state that will be realized in the distant future,

$$
\left|\Psi_{f}\right\rangle=S\left|\Psi_{i}\right\rangle
$$

The scattering matrix $T\left(\varepsilon_{f}, \varepsilon_{i}\right)$, defined as the probability per unit final state energy that a particle of energy $\varepsilon_{i}$ incident from the left contact will be transmitted with energy $\varepsilon_{f}$ into the right contact, can therefore be written

$$
\begin{aligned}
T\left(\varepsilon_{f}, \varepsilon_{i}\right)= & \sum_{\alpha_{f}, \alpha_{i}} \frac{e^{-\beta E_{\alpha_{i}}}}{Z}\left|\left\langle\varepsilon_{f}, \alpha_{f}, R|S| \phi_{\varepsilon_{i}}, \alpha_{i}, L\right\rangle\right|^{2} \\
= & \int d \varepsilon^{\prime} \int d \varepsilon \frac{1}{\Delta \varepsilon} \phi^{*}\left[\frac{\varepsilon^{\prime}-\varepsilon_{i}}{\Delta \varepsilon}\right] \\
& \times \phi\left[\frac{\varepsilon-\varepsilon_{i}}{\Delta \varepsilon}\right] P\left(\varepsilon_{f}, \varepsilon^{\prime}, \varepsilon\right)
\end{aligned}
$$

where $E_{\alpha_{i}}$ is the energy of the phonon system in state $\alpha_{i}$, $Z$ is the phonon partition function, and

$$
\begin{gathered}
P\left(\varepsilon_{f}, \varepsilon^{\prime}, \varepsilon\right)=\sum_{\alpha_{f}, \alpha_{i}} \frac{e^{-\beta E_{\alpha_{i}}}}{Z}\left\langle\varepsilon_{f}, \alpha_{f}, R|S| \varepsilon^{\prime}, \alpha_{i}, L\right\rangle^{*} \\
\times\left\langle\varepsilon_{f}, \alpha_{f}, R|S| \varepsilon, \alpha_{i}, L\right\rangle .
\end{gathered}
$$

The weighted sum over initial phonon states serves to average the transmission probability over a thermal distribution of phonons. The lattice is therefore assumed to be in equilibrium as the incident electron approaches the tunneling structure.

One way of writing the $S$ matrix that leads naturally to a Green-function expression for the transmission matrix is $^{24}$

$$
\begin{aligned}
& S=-i \int_{-\infty}^{\infty} \frac{d t_{1}}{\hbar} e^{i H_{0} t_{1} / \hbar} H_{1} e^{-i H_{0} t_{1} / \hbar} e^{-\eta\left|t_{1}\right|} \\
&-i \int_{-\infty}^{\infty} \int_{-\infty}^{\infty} \frac{d t_{1} d t_{2}}{\hbar^{2}} e^{i H_{0} t_{2} / \hbar} H_{1} \widehat{G}_{R}\left(t_{2}-t_{1}\right) \\
& \\
& \quad \times H_{1} e^{-i H_{0} t_{1} / \hbar} e^{-\eta\left(\left|t_{1}\right|+\left|t_{2}\right|\right)}, \quad \eta \rightarrow 0^{+} .
\end{aligned}
$$

The one-particle Green-function operator for the resonant level,

$$
\hat{G}_{R}(t)=-i \Theta(t) e^{-i H t / \hbar},
$$

includes both tunneling into the contacts and the electron-phonon interaction. Only the last term in the $S$-matrix contributes to the transmission probability because it takes at least two factors of the interaction Hamiltonian $H_{1}$ to hop the particle from the left contact onto the resonant site and then into the right contact. Also, because the only piece of $H_{1}$ which acts on an electron in the contacts is hopping onto the resonant site, the bracketing factors of $H_{1}$ in the last term of $S$ must be hops, first out of the left contact and finally into the right contact. Therefore, the matrix element in $P\left(\varepsilon_{f}, \varepsilon^{\prime}, \varepsilon\right)$ between an initial state on the left of energy $\varepsilon$ and a final state on the right with energy $\varepsilon_{f}$ is

$$
\begin{aligned}
\left\langle\varepsilon_{f}, \alpha_{f}, R|S| \varepsilon, \alpha_{i}, L\right\rangle & =-i \iint \frac{d t_{1} d t_{2}}{\hbar^{2}} e^{-\eta\left(\left|t_{1}\right|+\left|t_{2}\right|\right)}\left\langle\varepsilon_{f}, \alpha_{f}, R\left|e^{i H_{0} t_{2} / \hbar} H_{1} \hat{G}_{R}\left(t_{2}-t_{1}\right) H_{1} e^{-i H_{0} t_{1} / \hbar}\right| \varepsilon, \alpha_{i}, L\right\rangle \\
& =-V_{R}\left(\varepsilon_{F}\right) V_{L}(\varepsilon) \iint \frac{d t_{2} d t_{1}}{\hbar^{2}} e^{-\eta\left(\left|t_{1}\right|+\left|t_{2}\right|\right)} e^{i\left(\varepsilon_{f} t_{2}-\varepsilon t_{1}\right)} \Theta\left(t_{2}-t_{1}\right)\left\langle 0, \alpha_{f}\left|c\left(t_{2}\right) c^{\dagger}\left(t_{1}\right)\right| 0, \alpha_{i}\right\rangle
\end{aligned}
$$

where $\left|V_{L(R)}(\varepsilon)\right|^{2}=\sum_{k}\left|V_{k L(k R)}\right|^{2} \delta\left(\varepsilon-\varepsilon_{k L(k R)}\right)$ properly includes the density of states in the contacts in the hopping 
matrix elements. The expectation value $\left\langle 0, \alpha_{f}\left|c\left(t_{2}\right) c^{\dagger}\left(t_{1}\right)\right| 0, \alpha_{i}\right\rangle$ is taken between states of phonon occupation $\alpha_{i}$ and $\alpha_{f}$ with no electron present. In general, the expectation value will be nonzero because the time evolution of the electron operators,

$$
c(t)=e^{i H t / \hbar} c(0) e^{-i H t / \hbar},
$$

includes the emission and absorption of phonons through the electron-phonon interaction in $H$.

Substituting the matrix elements of the $S$ matrix back into $P\left(\varepsilon_{f}, \varepsilon^{\prime}, \varepsilon\right)$, one finds

$$
\begin{aligned}
& P\left(\varepsilon_{f}, \varepsilon^{\prime}, \varepsilon\right)=V_{L}(\varepsilon) V_{L}^{*}\left(\varepsilon^{\prime}\right)\left|V_{R}\left(\varepsilon_{f}\right)\right|^{2} \iiint \int \frac{d s_{1} d s_{2} d t_{1} d t_{2}}{\hbar^{4}} e^{-\eta\left(\left|s_{1}\right|+\left|s_{2}\right|+\left|t_{1}\right|+\left|t_{2}\right|\right)} e^{i\left(\varepsilon^{\prime} s_{1}-\varepsilon_{f} s_{2}-\varepsilon t_{1}+\varepsilon_{f} t_{2}\right)} \Theta\left(t_{2}-t_{1}\right) \\
& \times \Theta\left(s_{2}-s_{1}\right)\left\langle c\left(s_{1}\right) c^{\dagger}\left(s_{2}\right) c\left(t_{2}\right) c^{\dagger}\left(t_{1}\right)\right\rangle,
\end{aligned}
$$

where the angular brackets indicate a state with no electron present and a thermal ensemble of the phonons. The expectation value apparently depends on four times, but must be unchanged by an overall time translation since the Hamiltonian is time independent. To remove the extra time dependence, make the change of variables,

$$
t_{0}=t_{1}, \quad t=t_{2}-t_{1}, \quad \tau=s_{2}-t_{1}, \quad s=s_{2}-s_{1} .
$$

The integration over $t_{0}$ now yields a $\delta$ function of energies as $\eta \rightarrow 0^{+}$,

$$
\begin{gathered}
P\left(\varepsilon_{f}, \varepsilon^{\prime}, \varepsilon\right)=V_{L}(\varepsilon) V_{L}^{*}\left(\varepsilon^{\prime}\right)\left|V_{R}\left(\varepsilon_{f}\right)\right|^{2} 2 \pi \delta\left(\varepsilon^{\prime}-\varepsilon\right) \\
\times \iiint \frac{d \tau d s d t}{\hbar^{3}} e^{i\left[\left(\varepsilon^{\prime}-\varepsilon_{f}\right) \tau+\varepsilon_{f} t-\varepsilon^{\prime} s\right]} \\
\times G(\tau, s, t),
\end{gathered}
$$

where the expectation value of electron operators has been identified as the Green function,

$G(\tau, s, t)=\Theta(s) \Theta(t)\left\langle c(\tau-s) c^{\dagger}(\tau) c(t) c^{\dagger}(0)\right\rangle$.

Substituting $P\left(\varepsilon_{f}, \varepsilon^{\prime}, \varepsilon\right)$ into expression (A6) for the transmission matrix $T\left(\varepsilon_{f}, \varepsilon_{i}\right)$, and letting the energy width of the incident wave packet go to zero,

$$
\frac{1}{\Delta \varepsilon}\left|\phi\left(\frac{\varepsilon-\varepsilon_{i}}{\Delta \varepsilon}\right)\right|^{2} \rightarrow \delta\left(\varepsilon-\varepsilon_{i}\right),
$$

yields

$$
\begin{aligned}
T\left(\varepsilon_{f}, \varepsilon_{i}\right)= & \Gamma_{L}\left(\varepsilon_{i}\right) \Gamma_{R}\left(\varepsilon_{f}\right) \\
& \times \iiint \frac{d \tau d s d t}{2 \pi \hbar^{3}} e^{i\left[\left(\varepsilon_{i}-\varepsilon_{f}\right) \tau+\varepsilon_{f} t-\varepsilon_{i} s\right] / \hbar} \\
& \times G(\tau, s, t),
\end{aligned}
$$

where

$$
\begin{aligned}
\Gamma_{L(R)}(\varepsilon) & =2 \pi\left|V_{L(R)}(\varepsilon)\right|^{2} \\
& =2 \pi \sum_{k}\left|V_{k L(k R)}\right|^{2} \delta\left(\varepsilon-\varepsilon_{k L(k R)}\right) .
\end{aligned}
$$

Equation (A17) is a formally exact expression for the transmission matrix, including electron-phonon interaction, in terms of a two-particle Green function of the resonant site. The familiar tools of Green-function analysis can now be employed to carry out the evaluation of $T\left(\varepsilon_{f}, \varepsilon_{i}\right)$.
One obtains a reflection matrix $R\left(\varepsilon_{f}, \varepsilon_{i}\right)$ defined in analogy to the transmission matrix, by taking the projection of the final state $\left|\Psi_{f}\right\rangle$ onto the left contact in Eq. (A6) instead of onto the right. The analysis is identical to the case of transmission with the one additional complication that the first term of the $S$ matrix cannot be neglected for the case of elastic backscattering. The result is

$$
\begin{aligned}
R\left(\varepsilon_{f}, \varepsilon_{i}\right)= & \frac{\Gamma_{L}\left(\varepsilon_{f}\right) T\left(\varepsilon_{f}, \varepsilon_{i}\right)}{\Gamma_{R}\left(\varepsilon_{f}\right)} \\
& +\delta\left(\varepsilon_{i}-\varepsilon_{f}\right)\left\{1+2 \Gamma_{L}\left(\varepsilon_{i}\right) \operatorname{Im}\left[G_{R}\left(\varepsilon_{i}\right)\right]\right\},
\end{aligned}
$$

where

$$
G_{R}(\varepsilon)=\int \frac{d t}{\hbar} e^{i \varepsilon t / \hbar}\left[-i \Theta(t)\left\langle c(t) c^{\dagger}(0)\right\rangle\right] .
$$

\section{APPENDIX B: SOLUTION OF $G(\tau, s, t)$ BY EXPONENTIAL RESUMMATION}

One method of solving the transmission Green function $G(\tau, s, t)$ in Eq. (14) is to resum the results of a loworder perturbative expansion in the electron-phonon coupling. While it is only in the wide-band limit that resummation provides an exact solution, the leading terms in a perturbative expansion are valid for any bandwidth in the leads or hopping matrix elements $V_{k L}$ and $V_{k R}$, provided the coupling to phonons is weak. We begin this Appendix by expanding $G(\tau, s, t)$ perturbatively to lowest order in the electron-phonon coupling, and then carry out the procedure of exponential resummation in the wide-band limit.

The perturbative expansion of the interacting Green function is a standard application of many-body perturbation theory, ${ }^{40}$

$$
\begin{aligned}
G(\tau, s, t)=\boldsymbol{\Theta}(s) \Theta(t) & \left\langle\hat{c}(\tau-s) U^{\dagger}(\tau, \tau-s)\right. \\
& \left.\times \widehat{c}^{\dagger}(\tau) \hat{c}(t) U(t, 0) \hat{c}^{\dagger}(0)\right\rangle,
\end{aligned}
$$

where the $\widehat{c}^{\prime} \mathbf{s}$ and $\hat{c}^{\dagger}$ 's are operators in the interaction picture,

$$
\widehat{c}(t)=e^{i H_{0} t / \hbar} c(0) e^{-i H_{0} t / \hbar},
$$

and $U\left(t, t_{0}\right)$ is the time-evolution operator, 


$$
U\left(t, t_{0}\right)=\sum_{m=0}^{\infty} T\left[-i \int_{t_{0}}^{t} d t^{\prime} \hat{H}_{\mathrm{int}}\left(t^{\prime}\right)\right]^{m} / m ! .
$$

In the Hermitian adjoint of the time-evolution operator $U^{\dagger}\left(t, t_{0}\right)$, the time-ordering operator $T$ becomes an antitime-ordering operator $T^{-1}$,

$$
U^{\dagger}\left(t, t_{0}\right)=\sum_{m=0}^{\infty} T^{-1}\left[i \int_{t_{0}}^{t} d t^{\prime} \hat{H}_{\mathrm{int}}\left(t^{\prime}\right)\right]^{m} / m ! .
$$

The bare Hamiltonian $H_{0}$ includes the tunneling matrix elements, so $\hat{H}_{\text {int }}$ contains only the electron-phonon interaction on the resonant site,

$$
\hat{H}_{\mathrm{int}}(t)=\hat{c}^{\dagger}(t) \hat{c}(t) \sum_{\mathrm{q}} M_{\mathrm{q}}\left[\hat{a}_{\mathrm{q}}^{\dagger}(t)+\hat{a}_{-\mathrm{q}}(t)\right],
$$

where

$$
\widehat{a}_{\mathrm{q}}(t)=a_{\mathrm{q}} e^{-i \omega_{\mathrm{q}} t} \text { and } \hat{a}_{\mathrm{q}}^{\dagger}(t)=a_{\mathrm{q}}^{\dagger} e^{i \omega_{\mathrm{q}} t} \text {. }
$$

The interaction Hamiltonian $\hat{H}_{\text {int }}$ is zero when there is no electron on the resonant site, so there is no timeevolution operator between $\hat{c}^{\dagger}(\tau)$ and $\hat{c}(t)$ in the expectation value in Eq. (B1).

Each term in the perturbation expansion of $G(\tau, s, t)$ in powers of $H_{\text {int }}$ can be represented by a diagram, as indicated in Fig. 9, where each solid line corresponds to an electron Green function and each vertex corresponds to one appearance of the electron-phonon interaction $H_{\mathrm{int}}$. A phonon line connecting two vertices at $t_{1}$ and $t_{2}$ therefore contributes a factor

$$
\sum_{\mathbf{q}}\left|M_{\mathbf{q}}\right|^{2}\left[N_{\omega_{\mathrm{q}}} e^{i \omega_{\mathbf{q}}\left(t_{2}-t_{1}\right)}+\left(1+N_{\omega_{\mathbf{q}}}\right) e^{-i \omega_{\mathbf{q}}\left(t_{2}-t_{1}\right)}\right],
$$

where $N_{\omega_{\mathrm{q}}}$ is the Bose-Einstein occupation factor for phonon mode q. The leading term, with no phonons present, is the Green function in the absence of electron-
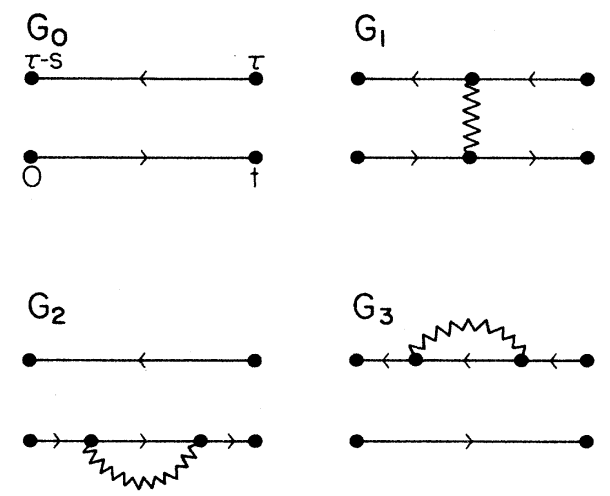

FIG. 9. Diagrammatic representation of the leading terms in a perturbative expansion of the interacting Green function $G(\tau, s, t)$. The first diagram $G_{0}(\tau, s, t)$ - the sole diagram in the absence of electron-phonon interaction - consists of two uncoupled single-particle Green functions. The second diagram $G_{1}(\tau, s, t)$, which has a single phonon line linking the two electronic Green functions, represents the emission or absorption of one real phonon. The last two diagrams $G_{2}(\tau, s, t)$ and $G_{3}(\tau, s, t)$ describe the propagation of an electron dressed by a virtual phonon.

phonon interaction,

$$
G_{0}(\tau, s, t)=G_{R}^{0}(t)\left[G_{R}^{0}(s)\right]^{*} .
$$

There are three separate terms which involve the electron-phonon interaction at second order; there are no first-order terms because in Eq. (B1) all phonon emissions must be balanced by phonon absorptions. The first interacting term represents the emission or absorption of one real phonon,

$$
\begin{aligned}
G_{1}(\tau, s, t)=\sum_{\mathrm{q}}\left|M_{\mathrm{q}}\right|^{2} & {\left[N_{\omega_{\mathrm{q}}} e^{i \omega_{\mathrm{q}} \tau} \int_{-\infty}^{\infty} d s^{\prime}\left[G_{R}^{0}\left(s-s^{\prime}\right)\right]^{*}\left[G_{R}^{0}\left(s^{\prime}\right)\right]^{*} e^{-i \omega_{\mathrm{q}}\left(s-s^{\prime}\right)} \int_{-\infty}^{\infty} d t^{\prime} G_{R}^{0}\left(t-t^{\prime}\right) G_{R}^{0}\left(t^{\prime}\right) e^{-i \omega_{\mathrm{q}} t^{\prime}}\right.} \\
& \left.+\left(1+N_{\omega_{\mathrm{q}}}\right) e^{-i \omega_{\mathrm{q}} \tau} \int_{-\infty}^{\infty} d s^{\prime}\left[G_{R}^{0}\left(s-s^{\prime}\right)\right]^{*}\left[G_{R}^{0}\left(s^{\prime}\right)\right]^{*} e^{i \omega_{\mathrm{q}}\left(s-s^{\prime}\right)} \int_{-\infty}^{\infty} d t^{\prime} G_{R}^{0}\left(t-t^{\prime}\right) G_{R}^{0}\left(t^{\prime}\right) e^{i \omega_{\mathrm{q}} t^{\prime}}\right]
\end{aligned}
$$

The second and third terms, which are obviously related, represent the change in the propagation of the electron due to the presence of a virtual phonon. The second term is given by

$G_{2}(\tau, s, t)=\left[G_{R}^{0}(s)\right]^{*} \sum_{\mathrm{q}}\left|M_{\mathrm{q}}\right|^{2} \int_{-\infty}^{\infty} d t_{2}^{\prime} \int_{-\infty}^{\infty} d t_{1}^{\prime} G_{R}^{0}\left(t-t_{2}^{\prime}\right) G_{R}^{0}\left(t_{2}^{\prime}-t_{1}^{\prime}\right) G_{R}^{0}\left(t_{1}^{\prime}\right)\left[N_{\omega_{\mathrm{q}}} e^{i \omega_{\mathrm{q}}\left(t_{2}^{\prime}-t_{1}^{\prime}\right)}+\left(1+N_{\omega_{\mathrm{q}}}\right) e^{-i \omega_{\mathrm{q}}\left(t_{2}^{\prime}-t_{1}^{\prime}\right)}\right]$,

and the third term is the complex conjugate of the second with the last two times interchanged,

$$
G_{3}(\tau, s, t)=\left[G_{2}(\tau, t, s)\right]^{*}
$$

For a weak electron-phonon coupling, the sum of the four terms represented by the diagrams in Fig. 9, will be a good approximation to the full interacting Green function $G(\tau, s, t)$. For a strong electron-phonon coupling, one can extend the results of low-order perturbation theory by a technique called, alternatively, exponential resummation, or the cumulant expansion, or the linkedcluster expansion. ${ }^{41}$ Instead, of approximating $G(\tau, s, t)$ by the first few terms obtained from the expansion of the 
evolution operators, one reinterprets these terms as the beginning of the expanded form of an exponential series for $G(\tau, s, t)$. Schematically, if a Green function $G_{0}(t)$ has an ordinary perturbation series in powers of an interaction $g$,

$$
G(t)=G_{0}(t)+\sum_{l=1}^{\infty} g^{l} F_{l}(t)
$$

then there may exist another expansion

$$
G(t)=G_{0}(t) \exp \left[\sum_{l=1}^{\infty} g^{l} W_{l}(t)\right]
$$

which converges much more rapidly than the original series. Once the $F(t)$ 's are known it is straightforward to calculate the $W(t)$ 's by matching the expansions order by order. The exponential form of the perturbation series will be rapidly convergent if the important high-order diagrams are well approximated by products of low-order diagrams. ${ }^{41}$ In our case, exponential resummation of the perturbative results yields

$$
\begin{aligned}
G(\tau, s, t) & \simeq G_{0}+G_{1}+G_{2}+G_{3} \\
& \simeq G_{0} e^{\left(G_{1}+G_{2}+G_{3}\right) / G_{0}} .
\end{aligned}
$$

In the wide-band limit, where the noninteracting Green function is an exponential,

$$
G_{R}^{0}(t)=-i \Theta(t) e^{\left(-i \varepsilon_{0}-\Gamma / 2\right) t / \hbar},
$$

the higher-order diagrams are given exactly by products of the three interacting diagrams in Fig. 9, so that the exponential resummation in Eq. (B13) is exact. In the wide-band limit, we find

$$
G_{1}(\tau, s, t)=G_{0}(\tau, s, t) \sum_{\mathbf{q}}\left|\frac{M_{\mathrm{q}}}{\hbar \omega_{\mathrm{q}}}\right|^{2}\left[-N_{\omega_{\mathrm{q}}} e^{i \omega_{\mathrm{q}} \tau}\left(1-e^{-i \omega_{\mathrm{q}} s}\right)\left(1-e^{-i \omega_{\mathrm{q}} t}\right)-\left(1+N_{\omega_{\mathrm{q}}}\right) e^{-i \omega_{\mathrm{q}} \tau}\left(1-e^{i \omega_{\mathrm{q}} s}\right)\left(1-e^{i \omega_{\mathrm{q}} t}\right)\right],
$$

and

$$
G_{2}(\tau, s, t)=G_{0}(\tau, s, t)\left(i t \lambda-\sum_{\mathrm{q}}\left|\frac{M_{\mathrm{q}}}{\hbar \omega_{\mathrm{q}}}\right|^{2}\left[N_{\omega_{\mathrm{q}}}\left(1-e^{i \omega_{\mathrm{q}} t}\right)+\left(1+N_{\omega_{\mathrm{q}}}\right)\left(1-e^{-i \omega_{\mathrm{q}} t}\right)\right],\right.
$$

where $\lambda=\sum_{\mathrm{q}}\left(M_{\mathrm{q}}^{2} / \hbar \omega_{\mathrm{q}}\right)$. Recalling that $G_{3}(\tau, s, t)$ $=\left[G_{2}(\tau, t, s)\right]^{*}$, we substitute the above expressions into Eq. (B13) and obtain the solution for $G(\tau, s, t)$ given in Eq. (19).

\section{APPENDIX C: TRANSMISSION MATRIX $T\left(\varepsilon^{\prime}, \varepsilon\right)$ FOR EINSTEIN PHONONS AT $T=0 \mathrm{~K}$}

For the special case of an Einstein band of phonons with energy $\hbar \omega_{0}$, we derive a simple expression for the transmission matrix $T\left(\varepsilon^{\prime}, \varepsilon\right)$ in the wide-band limit. From Sec. II, Eq. (13), the interacting transmission matrix is given by

$$
\begin{gathered}
T\left(\varepsilon^{\prime}, \varepsilon\right)=\Gamma_{L}(\varepsilon) \Gamma_{R}\left(\varepsilon^{\prime}\right) \iiint \frac{d \tau d t d s}{2 \pi \hbar^{3}} e^{i\left[\left(\varepsilon-\varepsilon^{\prime}\right) \tau+\varepsilon^{\prime} t-\varepsilon s\right] / \hbar} \\
\times G(\tau, s, t),
\end{gathered}
$$

where

$$
G(\tau, s, t)=\Theta(s) \Theta(t)\left\langle c(\tau-s) c^{\dagger}(\tau) c(t) c^{\dagger}(0)\right\rangle .
$$

In the wide-band limit, in which the couplings to the contacts $\Gamma_{L}$ and $\Gamma_{R}$ are independent of energy, there exists an exact solution for the interacting Green function $G(\tau, s, t)$ [see Eq. (19)]. For the Einstein band at zero lattice temperature, we find

$$
G(\tau, s, t)=G_{R}^{0}(t)\left[G_{R}^{0}(s)\right]^{*} e^{i(t-s) \lambda / \hbar-g f(\tau, s, t)}
$$

with

$f(\tau, s, t)=2-e^{-i \omega_{0} t}-e^{i \omega_{0} s}+e^{-i \omega_{0} \tau}\left(1-e^{i \omega_{0} t}\right)\left(1-e^{i \omega_{0} s}\right)$,

where $G_{R}^{0}(t)$ is the noninteracting Green function of the resonant site,

$$
G_{R}^{0}(t)=-i \Theta(t) e^{\left(-i \varepsilon_{0}-\Gamma / 2\right) t / \hbar} .
$$

The electron-phonon interaction appears in Eq. (C3) via the coupling constant $g$, which depends on the electronphonon matrix elements $M_{\mathrm{q}}$ according to

$$
g=\sum_{\mathrm{q}}\left|M_{\mathrm{q}} / \hbar \omega_{0}\right|^{2} \text {. }
$$

The expression for $T\left(\varepsilon^{\prime}, \varepsilon\right)$ obtained by combining Eqs. (C1) and (C3) above can be simplified considerably. To begin, separate the integrals so that the $\tau$ integration can be performed first,

$$
\begin{aligned}
T\left(\varepsilon^{\prime}, \varepsilon\right)=\Gamma_{L} \Gamma_{R} e^{-2 g} \int_{0}^{\infty} \frac{d s}{\hbar} \exp \left\{\left[i\left(\varepsilon_{0}-\varepsilon-\lambda\right)-\Gamma / 2\right] s / \hbar+g e^{i \omega_{0} s}\right\} \\
\quad \times \int_{0}^{\infty} \frac{d t}{\hbar} F(s, t) \exp \left\{\left[-i\left(\varepsilon_{0}-\varepsilon-\lambda\right)-\Gamma / 2\right] t / \hbar+g e^{-i \omega_{0} t}-i\left(\varepsilon-\varepsilon^{\prime}\right) t / \hbar\right\}
\end{aligned}
$$


where

$$
\begin{aligned}
F(s, t)=\int_{-\infty}^{\infty} & \frac{d \tau}{2 \pi \hbar} e^{i\left(\varepsilon-\varepsilon^{\prime}\right) \tau / \hbar} \\
& \times \exp \left[-g e^{-i \omega_{0} \tau}\left(1-e^{i \omega_{0} t}\right)\left(1-e^{i \omega_{0} s}\right)\right] .
\end{aligned}
$$

Expanding the second exponential in $F(s, t)$ and integrating over $\tau$ yields

$$
\begin{aligned}
F(s, t)=\sum_{m=0}^{\infty} & \frac{g^{m}}{m !} e^{i m \omega_{0} t}\left[\left(1-e^{-i \omega_{0} t}\right)\left(1-e^{i \omega_{0} s}\right)\right]^{m} \\
& \times \delta\left(\varepsilon-\varepsilon^{\prime}-m \hbar \omega_{0}\right) .
\end{aligned}
$$

After interchanging the sum on $m$ with the two remaining integrals, the factor of $\exp \left(i m \omega_{0} t\right)$ in $F(s, t)$ cancels the factor of $\exp \left[-i\left(\varepsilon-\varepsilon^{\prime}\right) t / \hbar\right]$ in Eq. (C5), leaving the integrals as complex conjugates. The transmission matrix can therefore by compactly rewritten as

$$
\begin{aligned}
T\left(\varepsilon^{\prime}, \varepsilon\right)= & \Gamma_{L} \Gamma_{R} e^{-2 g} \\
& \times \sum_{m=0}^{\infty} \frac{g^{m}}{m !} \delta\left(\varepsilon-\varepsilon^{\prime}-m \hbar \omega_{0}\right)\left|D_{m}(\varepsilon)\right|^{2},
\end{aligned}
$$

where

$$
\begin{gathered}
D_{m}(\varepsilon)=\int_{0}^{\infty} \frac{d t}{\hbar}\left(1-e^{-i \omega_{0} t}\right)^{m} \\
\times \exp \left\{\left[-i\left(\varepsilon_{0}-\varepsilon-\lambda\right)-\Gamma / 2\right] t / \hbar\right. \\
\left.+g e^{-i \omega_{0} t}\right\}
\end{gathered}
$$

The relations

$$
\left(1-e^{-i \omega_{0} t}\right)^{m}=\sum_{j=0}^{m}(-1)^{j}\left[\begin{array}{c}
m \\
j
\end{array}\right) e^{-i j \omega_{0} t}
$$

and

$$
\exp \left(g e^{-i \omega_{0} t}\right)=\sum_{l=0}^{\infty} \frac{g^{l}}{l !} e^{-i l \omega_{0} t}
$$

permit the integral for $D_{m}(\varepsilon)$ to be turned into a sum,

$$
D_{m}(\varepsilon)=i \sum_{j=0}^{m}(1-)^{j}\left(\begin{array}{l}
m \\
j
\end{array}\right] \sum_{l=0}^{\infty} \frac{g^{l}}{l !}\left(\frac{1}{\left[\varepsilon-\left(\varepsilon_{0}-\lambda\right)-(j+l) \hbar \omega_{0}\right]+i \Gamma / 2}\right) .
$$

This is the expression that appears in Sec. II, Eq. (32).

*Present address: Department of Physics, Massachusetts Institute of Technology, Cambridge, MA 02139.

${ }^{1}$ A. D. Benoit, S. Washburn, C. P. Umbach, R. B. Laibowitz, and R. A. Webb, Phys. Rev. Lett. 57, 1765 (1986).

${ }^{2}$ M. Büttiker, Phys. Rev. Lett. 57, 1761 (1986).

${ }^{3}$ S. Maekawa, Y. Isawa, and H. Ebisawa, J. Phys. Soc. Jpn. 56, 25 (1987).

${ }^{4}$ H. U. Baranger, A. D. Stone, and D. P. DiVincenzo, Phys. Rev. B 37, 6521 (1988).

${ }^{5}$ C. L. Kane, P. A. Lee, and D. P. DiVincenzo, Phys. Rev. B 38, 7909 (1988)

${ }^{6}$ S. Hershfield and V. Ambegaokar, Phys. Rev. B 38, 7909 (1988).

${ }^{7}$ B. J. van Wees, H. van Houten, C. W. J. Beenakker, J. G. Williamson, L. P. Kouwenhoven, D. van der Marel, and C. T. Foxon, Phys. Rev. Lett. 60, 848 (1988).

${ }^{8}$ D. A. Wharam, T. J. Thornton, R. Newbury, M. Pepper, H. Ahmed, J. E. F. Frost, D. G. Hasko, D. C. Peacock, D. A. Ritchie, and G. A. C. Jones, J. Phys. C 21, L209 (1988).

${ }^{9}$ L. I. Glazman, G. B. Lesovik, D. E. Khmel'nitski, and R. I. Shekhter, Pis'ma Zh. Eksp. Teor. Fiz. 48, 218 (1988) [JETP Lett. 48, 238 (1988)].

${ }^{10}$ G. Kirczenow, Solid State Commun. 68, 715 (1988).

${ }^{11}$ A. Szafer and A. D. Stone, Phys. Rev. Lett. 62, 300 (1989).

${ }^{12}$ E. G. Haanappel and D. van der Marel, Phys. Rev. B 39, 5484 (1989).

${ }^{13}$ M. L. Roukes, A. Scherer, S. J. Allen, Jr., H. G. Craighead, R. M. Ruthen, E. D. Beebe, and J. P. Harbison, Phys. Rev. Lett. 59, 3011 (1987).
${ }^{14}$ H. U. Baranger and A. D. Stone, Phys. Rev. Lett. 63, 414 (1989).

${ }^{15}$ A. D. Stone and P. A. Lee, Phys. Rev. Lett. 54, 1196 (1985).

${ }^{16}$ M. Jonson and A. Grincwajg, Appl. Phys. Lett. 51, 1729 (1987).

${ }^{17}$ Hu Yuming, J. Phys. C 21, L23 (1988).

${ }^{18}$ Ned S. Wingreen, Karsten W. Jacobsen, and John W. Wilkins, Phys. Rev. Lett. 61, 1396 (1988).

${ }^{19}$ E. H. Hauge and J. A. Stoevneng (unpublished).

${ }^{20}$ L. I. Glazman and R. I. Shekhter, Solid State Commun. 66, 65 (1988).

${ }^{21}$ L. I. Glazman and R. I. Shekhter, Zh. Eksp. Teor. Fiz. 94, 292 (1987) [Sov. Phys. - JETP 67, 163 (1988)].

${ }^{22}$ M. Jonson, Phys. Rev. B 39, 5924 (1989).

${ }^{23}$ A. L. Fetter and J. D. Walecka, Quantum Theory of ManyParticle Systems (McGraw-Hill, New York, 1971), pp. 105-107.

24J. R. Taylor, Scattering Theory (Wiley, New York, 1972), pp. 158-159.

${ }^{25}$ D. C. Langreth, Phys. Rev. B 1, 471 (1970).

${ }^{26}$ C.-O. Ambladh and P. Minnhagen, Phys. Rev. B 17, 929 (1978).

${ }^{27}$ G. D. Mahan, Many-Particle Physics (Plenum, New York, 1981), pp. 269-310.

${ }^{28}$ G. D. Mahan, Phys. Rev. B 15, 4587 (1977).

${ }^{29}$ P. J. Price, Phys. Rev. B 38, 1994 (1988).

${ }^{30} \mathrm{G}$. Timp (private communication).

${ }^{31}$ P. M. Mankiewich, R. E. Behringer, R. E. Howard, A. M. Chang, T. Y. Chang, B. Chelluri, J. Cunningham, and G. 
Timp, J. Vac. Sci. Technol. B 6, 131 (1988); G. Timp, H. U. Baranger, P. deVegvar, J. E. Cunningham, R. E. Howard, R. Behringer, and P. M. Mankiewich, Phys. Rev. Lett. 60, 2081 (1988).

${ }^{32}$ G. D. Mahan, Many-Particle Physics (Plenum, New York, 1981), pp. 33-38.

${ }^{33}$ G. D. Mahan, Many-Particle Physics (Plenum, New York, 1981), pp. 42-45.

${ }^{34} \mathrm{M}$. Neuberger, Handbook of Electrical Materials, Vol. 2, III-V Semiconducting Compounds (IFI/Plenum, New York, 1971), p. 47.

${ }^{35}$ S. S. Devlin, in Physics and Chemistry of II-VI Compounds, edited by M. Aven and J. S. Prener (North-Holland, Amster- dam, 1967), p. 603.

${ }^{36}$ L. D. Landau and E. M. Lifshitz, Quantum Mechanics (Pergamon, New York, 1977), pp. 76-78.

${ }^{37}$ M. Cahay, M. McLennan, S. Datta, and M. S. Lundstrom, Appl. Phys. Lett. 50, 612 (1987).

${ }^{38}$ V. J. Goldman, D. C. Tsui, and J. E. Cunningham, Phys. Rev. Lett. 58, 1256 (1987).

${ }^{39} \mathrm{~S}$. Ralph and F. Capasso (private communication).

${ }^{40}$ A. L. Fetter and J. D. Walecka, Quantum Theory of ManyParticle Systems (McGraw-Hill, New York, 1971), pp. 54-58.

${ }^{41}$ G. D. Mahan, Many-Particle Physics (Plenum, New York, 1981), pp. 183-185. 